\title{
The galaxy cluster Abell 426 (Perseus). A catalogue of 660 galaxy positions, isophotal magnitudes and morphological types ${ }^{\star, \star \star}$
}

\author{
J. Brunzendorf ${ }^{\star \star \star}$ and H. Meusinger ${ }^{\star \star \star}$ \\ Thüringer Landessternwarte Tautenburg, Sternwarte 5, D- 07778 Tautenburg, Germany \\ e-mail: brunz@tls-tautenburg.de/meus@tls-tautenburg.de
}

Received March 3; accepted July 1, 1999

\begin{abstract}
We present a homogeneous catalogue of galaxies in the field of the nearby galaxy cluster A 426 (Perseus) based on a survey of digitised Schmidt plates taken with the Tautenburg $2 \mathrm{~m}$ telescope in the $B$ band. Accurate positions, morphological types, $B_{25}$ isophotal magnitudes, angular radii and position angles are given for 660 galaxies within a field of about 10 square-degrees, centred on $\alpha=3^{\mathrm{h}} 21^{\mathrm{min}}, \delta=41^{\circ} 33^{\prime}$ (J2000). When available, the radial velocity and the most common name taken from NED or PGC are included. The catalogue comprises galaxies brighter than $B_{25} \approx 19.5$. The estimated limit of completeness is $B_{25} \approx 18$. Two thirds of the galaxies are published for the first time. The galaxy positions are measured with a mean accuracy of 0.5 , the photometric accuracy is of the order of 0.1 to $0.2 \mathrm{mag}$ depending on image crowding and galaxy shape. Morphological properties were evaluated from the visual inspections of both deep images obtained from the digital co-addition of a large number of plates and higher-resolution images from single plates taken under good seeing conditions. The superimposed images unveil faint structures down to $\mu_{B} \approx 27 \mathrm{mag} \operatorname{arcsec}^{-2}$.
\end{abstract}

The catalogue is applied to a study of statistical properties of the galaxies in A 426: projected distribution of morphological types, segregation of morphological types, position of the cluster centre, distribution of galaxy position angles, type-dependent luminosity functions, and total $B$-luminosity of the the cluster. In agreement with

\footnotetext{
* The catalogue is only available in electronic form at the CDS via anonymous ftp to cdsarc.u-strasbg.fr (130.79.128.5) or via http://cdsweb.u-strasbg.fr/Abstract.html

** Based on observations made with the $2 \mathrm{~m}$ telescope of the Thüringer Landessternwarte Tautenburg, Germany, and with the $2.2 \mathrm{~m}$ telescope of the German-Spanish Astronomical Centre, Calar Alto, Spain.

$\star \star \star$ Visiting Astronomer, German-Spanish Astronomical Centre, Calar Alto, operated by the Max-Planck-Institute for Astronomy, Heidelberg, jointly with the Spanish National Comission for Astronomy.
}

previous studies, we find a relative spiral-deficiency in the central region $\left(r \lesssim 30^{\prime}\right)$. The percentage of identified $\mathrm{S}+$ Irr increases, however, increases from $30 \%$ in the centre to more than $50 \%$ in the outer parts. The projected distributions of early- and late-type galaxies are not co-centred. The total luminosity of all supposed member galaxies in the surveyed area is estimated to $(6.5 \pm 0.9) 10^{12} \times h_{50}^{-2}$ blue solar luminosities. We do not analyse in detail possible substructures in the projected distribution of galaxies. However, we found a pronounced clump of galaxies at $\alpha(\mathrm{J} 2000.0)=3^{\mathrm{h}} 20^{\mathrm{m}} 4, \delta(\mathrm{J} 2000.0)=43^{\circ} 4^{\prime}$, which is shown to be a background cluster at $z \approx 0.050$.

Key words: galaxies: clusters: individual: A 426 - galaxies: fundamental parameters - galaxies: luminosity function

\section{Introduction}

Rich galaxy clusters represent the largest bound masses in the present-day Universe. Differences in the properties of clusters, such as shape, substructure, galaxy concentration and galaxy population are generally thought to reflect different evolutionary stages. Hence, their investigation is important for the understanding of the formation and evolution of large aggregates in the Universe.

A 426 (Perseus) is a nearby rich galaxy cluster of Bautz-Morgan class II-III (Bautz \& Morgan 1970) and Rood-Sastry type L (Struble \& Rood 1987). It has been recognised long ago that this cluster is remarkable in several aspects: it shows one of the highest velocity dispersion among the nearby clusters, a prominent chain of bright galaxies in the core, and an exceptionally strong deficiency of spiral galaxies (Chincarini \& Rood 1971; Melnick \& Sargent 1977; Kent \& Sargent 1983). Recent studies, however, find a higher fraction of spirals (Andreon 1994). A 426 is one of the brightest clusters observed in the 
X-ray waveband with a massive cooling flow in its centre (Mushotsky et al. 1978; Allen et al. 1992; Allen \& Fabian 1997; Peres et al. 1998). X-ray emission has been observed up to more than one degree from the cluster core (Nulsen \& Fabian 1980; Ulmer et al. 1980; Ettori et al. 1998). The $\mathrm{X}$-ray centre is not identical with the centre of the optical galaxy positions (Branduardi-Raymont et al. 1981; Snyder et al. 1990; Ulmer et al. 1992) which has been interpreted as a signature of a cluster merger (Ulmer et al. 1992). Significant substructure in the X-ray image has been discussed as evidence that the cluster is not in a relaxed state and is consistent with a recent merger (Schwarz et al. 1992; Mohr et al. 1993; Ettori et al. 1998). Clear evidence for substructure in the optical galaxy distribution has been reported in several studies (Baier 1979, 1984; Escalara et al. 1994, Andreon 1994) whereas others did not find strong substructure (Geller \& Beers 1982; Fitchett \& Webster 1987; Dressler \& Shectman 1988) or no substructure at all (Bird 1994). The Perseus cluster hosts several galaxies with active nuclei, pronounced radio structures, enhanced far-infrared emission, and/or signs of strong gravitational interactions.

The complex innermost central region of the cluster including the remarkable central dominant galaxy NGC 1275 has been the subject of various detailed studies. NGC 1275 hosts an active nucleus with spectral and radio properties intermediate between a Seyfert galaxy and a BL Lac object (Ferruit \& Pécontal 1994; Véron 1978). The nucleus is associated with the FR I radio source Per A which has been intensively studied on different scales (e.g. Ryle \& Windram 1968; Pedlar et al. 1990; Vermeulen et al. 1994). The lobes of Per A are interacting with the X-ray emitting intra-cluster plasma, and their structure may be explained as due to an oscillating motion of the cD galaxy NGC 1275 in the cluster potential (Böhringer et al. 1993). The radio lobes are embedded within a radio halo which has been traced out to a diameter of $300 \mathrm{kpc}$ (Pedlar et al. 1990; Burns et al. 1992). NGC 1275 is surrounded by an extended system of optical emission-line filaments with a complex velocity structure (Minkowski 1957; Burbidge \& Burbidge 1965; Rubin et al. 1977; Kent \& Sargent 1979; Caulet et al. 1992; Ferruit et al. 1997). The low velocity filament system has been related either to the cooling flow (Fabian \& Nulsen 1977; Fabian 1994; McNamara et al. 1996; Dixon et al. 1996) or to the occurrence of a recent merger which was also suggested to explain the origin of the system of young globular clusters (Holtzman et al. 1992).

Due to its proximity and its richness, A 426 is well suited for the investigation of several aspects of the structure of galaxy clusters and statistical properties of cluster galaxies, such as luminosity and morphological segregation or luminosity functions. On the other hand, however, such studies are hampered by the low Galactic latitude of the field (the Perseus cluster is located at $l=150^{\circ} 6, b=-13.3$ ) and, consequently, by the high density of Galactic foreground stars as well as increased, and perhaps irregular, foreground extinction. This may be one reason why the optical data base for the galaxies in A 426 is relatively poor compared with other nearby clusters. (For instance, for the Coma cluster Doi et al. (1995) presented homogeneous photometric data and morphology for 450 galaxies, and the "Virgo photometry catalogue" (Young \& Currie 1998) contains 1180 galaxies.) Chincarini \& Rood (1971) based their study of the dynamics of A 426 on 47 galaxies. Melnick \& Sargent (1977) classified 175 galaxies brighter than $m_{\mathrm{pg}} \approx 16$ in a region of about $100^{\prime}$ from the cluster centre on a Palomar IIIa-J Schmidt plate. They found an exceptionally small number of spirals (7\%) with a flat radial distribution to be compared with a steep radial distribution for S0s and Es. Bucknell et al. (1979) presented photographic photometry for 233 galaxies with $V_{25}<17.5$ in the inner region of A 426 within a cluster-centric radius of $\approx 30^{\prime}$. Galaxies of this "BGP sample" were combined by Kent \& Sargent (1983, hereafter KS 83) with a nearcomplete sample of galaxies brighter than $m_{\mathrm{pg}} \approx 15.7$ within a radius of $3^{\circ}$ from NGC 1275 taken from the Catalogue of Galaxies and Clusters of Galaxies (Zwicky \& Kowal 1968), hereafter called "Zwicky sample". A combined sample of 190 galaxies was used by KS 83 for their study of the dynamics of the Perseus cluster.

Morphological types, estimated from a Palomar Schmidt plate, have been available only for a small fraction of the galaxies from the KS 83 sample. Significantly refined morphological information were derived by Poulain et al. (1992) for 91 early-type galaxies of this list from a detailed isophotal shape analysis. Thanks to the high resolution of their CCD images, a variety of fine details in the structure of the galaxy images could be uncovered. These results have demonstrated the sensitivity of recognising the morphological types for rather remote galaxies on the detectability of fine structure details, i.e. on the signal-to-noise ratio and spatial resolution. In particular, the revision of the morphological types has drastically decreased the estimated percentage of Es and S0s by a factor of more than two. Andreon (1994) studied morphological segregations in A 426 from a BGP subsample of 96 galaxies with $B \leq 15.7$ in the inner $\sim 30^{\prime}$ region. He took the morphological type information from Poulain et al. (1992) for most galaxies and from a visual inspection of Schmidt plates taken at Palomar and Calern, respectively, in the remaining cases. Andreon et al. (1997a) extended the isophotal analysis and provided morphological data for a complete sample of galaxies with $m_{\text {Zwicky }}<15.7$ in a region of $5.3 \times 5.27$. De Propris \& Pritchet (1998) presented a study of the faint end of the galaxy luminosity function from a deep $14^{\prime} \times 14^{\prime}$ image of the cluster centre taken with the $3.6 \mathrm{~m}$ CFHT in the $I$-band.

The main aim of the present paper is to contribute to an extended data base for the galaxies in a large field of 
the Perseus cluster. We provide a new catalogue of 660 galaxies in a field of nearly 10 square degrees containing accurate positions (important for cross-identifications), photometric data and morphological information. Two thirds of the galaxies are published for the first time. The study is based on digitised Schmidt plates taken with the Tautenburg $2 \mathrm{~m}$ telescope. Schmidt plates still provide an important tool for the investigation of clusters with extentions as large as for A 426. The disadvantage of a lower limiting magnitude, as compared with deep CCD images, can be widely reduced by the digital co-addition of a large number of plates. Due to the gain in the signal-tonoise ratio, stacking of Schmidt plates provides a powerful method for the evaluation of faint, extended structure details. Preliminary results from this study have been discussed in Brunzendorf \& Meusinger (1996).

The observational material used for the present study is described in the next Section. The digitisation of the Schmidt plates by means of the Tautenburg plate scanner is outlined in Sect. 3, followed by a description of the procedures for obtaining galaxy positions, photometric information, and some radial velocities in Sect. 4 . Section 5 deals with the evaluation of morphological properties. The structure of the galaxy catalogue is briefly described in Sect. 6. Finally, the catalogue data are used in Sect. 7 to demonstrate several statistical properties of the galaxies in A 426: the projected distributions of morphological types, the position of the cluster centre, morphological segregation, the distribution of the galaxy position angles, the $B$-band luminosity function, and the total $B$-luminosity of the cluster. Conclusions are given in Sect. 8. The catalogue of galaxies in A 426 is only available in electronic form at the CDS.

Throughout this paper we assume a redshift of $z=$ 0.0183 (Abell et al. 1989) for the Perseus cluster. Angular separations can be converted into linear distances by $1^{\prime} \equiv 31.5 h_{50}^{-1} \mathrm{kpc}$.

\section{Observations}

\subsection{Schmidt plates}

In the framework of a recent supernova search program (Meusinger \& Brunzendorf 1996; Meusinger et al. 1999a), 30 plates have been taken with the Tautenburg $2 \mathrm{~m}$ telescope in its Schmidt mode $(134 \mathrm{~cm}$ free aperture, $4 \mathrm{~m}$ focal length) between September 1992 and March 1995. These plates provide, in combination with 7 older archive plates, the major part of the observational material for the present study (Table 1). The plate emulsions Kodak 103 a$\mathrm{O}$ as well as the similar ORWO AS and ORWO ZU 21 are sensitive in the blue spectral range. In combination with a Schott GG 13 filter, they closely match the Johnson $B$ band.

The plates cover an unvignetted area of $3.3 \times 3.3$ around the centre of the Perseus cluster at a scale of
Table 1. Summary of observations

\begin{tabular}{|c|c|c|c|c|}
\hline \multirow{2}{*}{$\begin{array}{c}\text { Plate } \\
\text { No. }{ }^{1}\end{array}$} & \multicolumn{2}{|c|}{ Plate Centre } & \multirow[b]{2}{*}{ Date } & \multirow[b]{2}{*}{ Emul.+Filter } \\
\hline & R.A. & Dec. & & \\
\hline 578 & $03: 21: 31$ & $+41: 06$ & 1962 SEP 28 & $103 \mathrm{a}-\mathrm{O}+\mathrm{GG} 13$ \\
\hline 591 & $03: 21: 25$ & $+41: 10$ & 1962 SEP 30 & $103 a-O^{2}$ \\
\hline 611 & $03: 21: 22$ & $+41: 11$ & 1962 OCT 02 & 103a-O+GG 13 \\
\hline 720 & 03:21:30 & $+41: 11$ & 1962 NOV 19 & $103 \mathrm{a}-\mathrm{O}^{2}$ \\
\hline 739 & $03: 21: 25$ & $+41: 11$ & 1962 DEC 02 & $\mathrm{AS}^{2}$ \\
\hline 6900 & 03:19:10 & $+41: 28$ & 1988 DEC 07 & ZU $21+$ GG 13 \\
\hline 6902 & 03:19:10 & $+41: 28$ & 1988 DEC 07 & $\mathrm{ZU} 21+\mathrm{GG} 13$ \\
\hline 8158 & $03: 19: 47$ & $+41: 30$ & 1992 SEP 27 & ZU $21+$ GG 13 \\
\hline 8184 & $03: 19: 47$ & $+41: 30$ & 1992 SEP 29 & ZU $21+$ GG 13 \\
\hline 8192 & $03: 19: 47$ & $+41: 30$ & 1992 OCT 24 & ZU $21+$ GG 13 \\
\hline 8211 & $03: 19: 47$ & $+41: 30$ & 1992 NOV 01 & ZU $21+$ GG 13 \\
\hline 8220 & 03:19:47 & $+41: 30$ & 1992 NOV 02 & ZU $21+$ GG 13 \\
\hline 8229 & $03: 19: 47$ & $+41: 30$ & 1992 NOV 24 & ZU $21+$ GG 13 \\
\hline 8253 & 03:19:47 & $+41: 30$ & 1993 JAN 02 & ZU $21+$ GG 13 \\
\hline 8256 & $03: 19: 47$ & $+41: 30$ & 1993 FEB 22 & ZU $21+$ GG 13 \\
\hline 8266 & $03: 20: 27$ & $+41: 33$ & 1993 MAR 19 & ZU 21+GG 13 \\
\hline 8382 & $03: 20: 27$ & $+41: 33$ & 1993 AUG 16 & ZU $21+$ GG 13 \\
\hline 8416 & $03: 20: 27$ & $+41: 33$ & 1993 SEP 22 & ZU $21+$ GG 13 \\
\hline 8417 & $03: 20: 27$ & $+41: 33$ & 1993 OCT 15 & ZU $21+$ GG 13 \\
\hline 8429 & $03: 20: 27$ & $+41: 33$ & 1993 OCT 18 & ZU $21+$ GG 13 \\
\hline 8446 & $03: 20: 27$ & $+41: 33$ & 1993 NOV 12 & ZU 21+GG 13 \\
\hline 8464 & $03: 20: 27$ & $+41: 33$ & 1993 NOV 18 & ZU $21+$ GG 13 \\
\hline 8490 & $03: 20: 27$ & $+41: 33$ & 1994 JAN 17 & ZU $21+$ GG 13 \\
\hline 8551 & $03: 20: 27$ & $+41: 33$ & 1994 MAR 10 & ZU $21+$ GG 13 \\
\hline 8715 & 03:20:30 & $+41: 33$ & 1994 AUG 14 & ZU $21+$ GG 13 \\
\hline 8719 & 03:21:10 & $+41: 33$ & 1994 AUG 15 & ZU $21+$ GG 13 \\
\hline 8737 & $03: 21: 10$ & $+41: 33$ & 1994 SEP 04 & ZU $21+$ GG 13 \\
\hline 8753 & 03:21:10 & $+41: 33$ & 1994 SEP 14 & ZU $21+$ GG 13 \\
\hline 8764 & 03:21:10 & $+41: 33$ & 1994 OCT 05 & ZU $21+$ GG 13 \\
\hline 8776 & 03:21:10 & $+41: 33$ & 1994 ОCТ 07 & ZU $21+$ GG 13 \\
\hline 8788 & 03:21:10 & $+41: 33$ & 1994 OCT 11 & ZU $21+$ GG 13 \\
\hline 8802 & 03:21:10 & $+41: 33$ & 1994 NOV 01 & ZU $21+$ GG 13 \\
\hline 8814 & $03: 21: 10$ & $+41: 33$ & 1994 NOV 02 & ZU $21+$ GG 13 \\
\hline 8841 & 03:21:10 & $+41: 33$ & 1995 JAN 30 & ZU $21+$ GG 13 \\
\hline 8868 & 03:21:10 & $+41: 33$ & 1995 FEB 22 & ZU $21+$ GG 13 \\
\hline 8885 & $03: 21: 25$ & $+41: 33$ & 1995 MAR 02 & ZU $21+$ GG 13 \\
\hline 8904 & 03:21:10 & $+41: 33$ & 1995 MAR 23 & ZU $21+$ GG 13 \\
\hline
\end{tabular}

${ }^{1}$ Numbers of plates selected for photometry and/or astrometry are printed italic. ${ }^{2}$ No filter used.

51.4 arcsec $\mathrm{mm}^{-1}$. With a typical exposure time of $30 \mathrm{~min}$ $(22-60 \mathrm{~min})$, the mean limiting magnitude is $B \approx 20$. The deepest plates have limits of $B \approx 21.5$. The mean seeing conditions were rather poor $\left(2^{\prime \prime}-3^{\prime \prime}\right)$. This is, however, not critical for the investigation of extended features in galaxies. On the other hand, the brighter fine structures, especially in the central regions of galaxies, can be studied well on those few plates taken under good seeing conditions $\left(\approx 1^{\prime \prime}\right)$. Almost every plate (except the oldest ones) has a calibration wedge in its northwestern corner.

\subsection{Low-dispersion spectroscopy}

Comprehensive radial velocity data are important in galaxy cluster studies in several respects. Our work, however, is not focused on a substantial enlargement of the radial velocity data base. Nonetheless, we were interested in further radial velocity measurements in the context of two particular issues: the substructure in the projected galaxy distribution 1.5 north of the cluster 
centre (see Sect.7.1) and the properties of the IRAS galaxies in the field (Meusinger \& Brunzendorf, in preparation). In the framework of the follow-up spectroscopy of QSO candidates in a different field (Meusinger et al. 1999b) we were in the position to use some observing time during the dawn for obtaining several low-dispersion spectra for five galaxies from our catalogue, namely two IRAS galaxies $(342,540)$ and three galaxies from the clump north of the cluster centre $(329,335,353)$. The observations were taken on July 26 and 27, 1998 with CAFOS at the $2.2 \mathrm{~m}$ telescope on Calar Alto, Spain, equipped with the B-400 grism and a SITe1d CCD. A slit of 1 .' 2 (July 26) or 1.'6 (July 27) yielded a resolution of about 20 or $30 \AA$, respectively. Two spectra were obtained for each galaxy with total exposure times between $370 \mathrm{~s}$ and $600 \mathrm{~s}$.

\section{Plate measurements}

\subsection{The Tautenburg Plate Scanner}

The Schmidt plates were completely digitised using the Tautenburg Plate Scanner (hereafter TPS). The TPS is a new fast plate measuring machine at the Thüringer Landessternwarte Tautenburg coming into operation during the last years. In the following, we shall briefly outline the basic concept and the main operational details of the TPS. A short description of the astrometric properties is given by Brunzendorf \& Meusinger (1998).

The main components of the TPS comprise (1.) a movable $X-Y$ plate carriage, (2.) a diffuse illumination screen below, and (3.) a direct CCD imaging system above the plate carriage. The TPS digitises plates by moving the plate through the optical path of the stationary imaging system. Plates up to $30 \mathrm{~cm} \times 30 \mathrm{~cm}$ can be measured.

The $X-Y$ plate carriage consists of two motor/encoder/stage units which permit independent motions along the $X$ and $Y$ directions. Prior to each scan, the $X-Y$ carriage is moved into an appropriate position. The $Y$ stage accelerates until it reaches the final velocity that will be kept throughout the scan. The begin of the data acquisition is triggered when the $Y$ stage reaches the actual start position. The rms positional repeatability of each stage is $0.4 \mu \mathrm{m}$. The stages have absolute systematic errors of up to $4 \mu \mathrm{m}(\mathrm{rms}=2 \mu \mathrm{m})$ with a scale length of $\sim 10 \mathrm{~cm}$. Currently, the stages are being upgraded with two linear encoders having an absolute accuracy better than $0.5 \mu \mathrm{m}$ over $30 \mathrm{~cm}$.

The part of the plate to be measured is backsideilluminated by a Fostec cold surface light source powered by a regulated $150 \mathrm{~W}$ tungsten-halogen lamp via optical fibres. The lamp brightness is electronically stabilized within better than $1 \%$. In addition, its actual flux is permanently monitored by means of a fibre optics that feeds light from the lamp directly onto a fraction of the detector array of the CCD (see below). This reference signal is used to correct the incoming data for remaining fluctuations of the lamp intensity. In this way, a photometric stability of better than $0.1 \%(0.0004 D)$ over $24 \mathrm{~h}$ is achieved.

A strip of the illuminated plate area is projected onto a $6000 \times 1$ photosite array (CCD 191 from Fairchild Weston Systems, Inc.) by a telecentric mapping lens system with unit magnification. The optics has a linear field diameter in the object plane of $60 \mathrm{~mm}$, a numerical aperture of 0.1 , and a focal depth of $30 \mu \mathrm{m}$. The telecentric projection has the important advantage of being less susceptible to scattered light, because any light transmitted through the emulsion contributes to forming a correct image of the plate, regardless of the origin of that light (e.g., Hambly et al. 1998; Miller et al. 1992).

The size of each CCD pixel is $10 \mu \mathrm{m} \times 10 \mu \mathrm{m}$, corresponding to $0.5 \times 0.5$ on a Tautenburg Schmidt plate. Because 500 pixels are reserved for the reference signal (see above), the effective width of the measured strip on the plate amounts to $55 \mathrm{~mm}$. The CCD is operated in a continuous scan mode, i.e. the photon-generated electrons are accumulated and read out periodically. The analogue output signal is linearly amplified and fed to a 12 bit $(=4096$ grey levels) analogue-to-digital converter. Thereafter, the digital signal is corrected for temporal light source intensity fluctuations (see above) and for spatial inhomogeneities of both the illumination system and the CCD pixel sensitivities ("flat field correction"). The final peakto-digitisation noise ratio of the TPS data amounts to $\geq 1000$. The obtained data are stored in a 16-bit FITS format file on the control computer. Optionally, the plate can be measured with reduced intensity resolution ( $8 \mathrm{bit}$ ).

Before a scan is started, the optimal CCD integration time and the camera focus are to be determined. The integration time is adjusted to the transmittance of the plate background corresponding to a fraction of 0.75 of the maximum CCD intensity range. Automated focussing is performed at 8 different positions on the plate; the median focus value is adopted for the whole scan. Thanks to the telecentric projection (telecentric depth $\approx 1 \mathrm{~cm}$ ) the astrometric and photometric bias caused by small focus deviations over the plate is negligible.

After every $4 \mathrm{~cm}$, the scanning process is interrupted for saving the data from RAM onto disk. This scanning interval is well-defined; it causes a random shift of the order of $1 \mu \mathrm{m}$ in the $Y$ position of all objects within one scanning section with respect to the neighbouring sections. This shift can be easily evaluated from the positions of the objects in the overlap region of neighbouring strips.

In general, plates are completely scanned in a series of overlapping lanes ("strip mode") by means of fully automatized control software. Alternatively, a number of subareas defined by a list of plate coordinates and area sizes can be measured ("batch mode"). A $24 \mathrm{~cm} \times 24 \mathrm{~cm}$ Tautenburg Schmidt plate is digitised within typically less than three hours. 
The scanner is operated by a DOS-PC with a Pentium $100 \mathrm{CPU}, 64 \mathrm{MB}$ RAM and two $2 \mathrm{~GB}$ hard disks for temporary storage of the incoming pixel data. Complete data files of the scanned plates are stored on DAT or CD-ROM. Final data reduction is done on remote workstations.

\subsection{Plate digitisation and selection of galaxies}

The 37 plates selected for further reduction (Table 1) were digitised in the "strip mode" with overlaps of $10 \mathrm{~mm}(8.6)$ between adjacent strips. Thanks to those overlaps, complete, non-truncated images are available for all galaxies. In a first run, digitisation was done in the 8 bit mode. Additionally, five selected plates were digitised later in the 12 bit mode for photometric and astrometric purposes (see below).

The two deepest digitised plates (Nos. 8753 and 8788), taken under good seeing conditions $\left(1^{\prime \prime}-1^{\prime \prime} .5\right)$, were independently surveyed by eye for galaxies. Only nonstellar objects found on both plates were selected. The final sample consists of 660 galaxies. The selection procedure may be biased against faint low surface brightness (LSB) galaxies as well as against very compact galaxies. Nevertheless, such a "by eye" selection method is expected to be more complete than usual automatic object classification methods (cf. O'Neil et al. 1997, for the case of LSB galaxies).

On each digitised plate, the images of all 660 galaxies were extracted into separate frames of $200^{\prime \prime} \times 200^{\prime \prime}$ size. For the more extended giant galaxy NGC 1275, a larger frame of $400^{\prime \prime} \times 400^{\prime \prime}$ was used. Each frame is centred on the deduced preliminary position of the galaxy's core.

\section{Data reduction}

\subsection{Galaxy positions}

Precise galaxy positions were independently derived from four deep plates (Nos. 8417, 8753, 8776 and 8788) taken under $1^{\prime \prime}-2^{\prime \prime}$ seeing conditions and digitised in the 12 bit mode. We usually applied the Gaussian centring algorithm of the standard MIDAS software package onto the galaxy cores. In a few doubtful cases of irregular galaxies or blended cores the centre position was estimated by eye. The reference frame for the astrometric reduction was realized using the 40 faintest stars from the PPM catalogue (Röser \& Bastian 1991) in this field. Each plate was separately modelled by a 2 nd order $2 \mathrm{D}$ polynomial fit. For each galaxy, the obtained four positions were averaged, yielding the mean galaxy position with a typical standard deviation $\sigma$ of 0.25 . The mean total error of the derived galaxy positions (including possible systematic ones) is expected to be $\leq 0^{\prime \prime} .5$.

We compare the obtained galaxy positions with the data from the Reference Galaxy List of the Lick Northern Proper Motion program (NPM, Klemola et al. 1987),

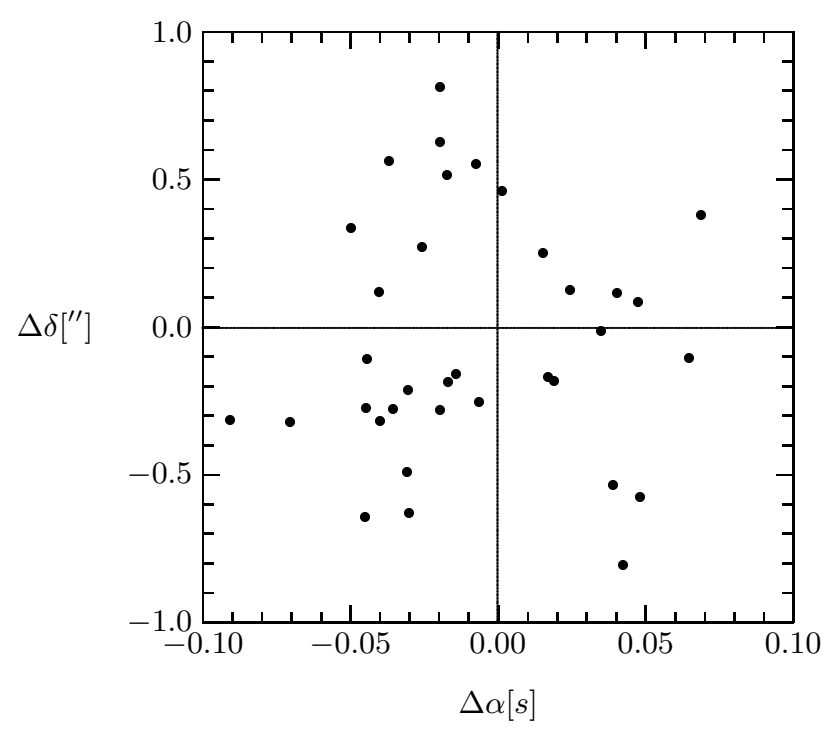

Fig. 1. Comparision of galaxy positions derived in this paper versus NPM values (Klemola et al. 1987). The plot shows the residuals in right ascension $\alpha$ and declination $\delta$ for all 35 galaxies common in both catalogues. The mean positional difference is 0.56

which contains 50517 galaxy positions with a typical rms error of $0 . \prime 3$. There are 35 NPM galaxies in our field, all of them could be cross-identified with galaxies of our survey. The differences between the NPM positions and ours are shown in Fig. 1; the mean residual is $00^{\prime \prime} 56$, none exceeds $1^{\prime \prime}$.

\subsection{Photometry}

Our photometry is based on the those four plates mentioned in Sect.4.1 in combination with plate 8841. The availability of five plates simplifies the plate fault removal and increases the signal-to-noise ratio. Again, subframes of $200^{\prime \prime} \times 200^{\prime \prime}$ have been extracted which are centred on the galaxy cores $\left(400^{\prime \prime} \times 400^{\prime \prime}\right.$ for NGC 1275$)$. Surface photometry is carried out according to the standard procedures (e.g. Okamura 1988):

The measured relative photographic transmission $T$ is converted into the relative intensity $I$ of the incident light by means of the characteristic curve which was approximated by

$\log _{10} I=c_{1}+c_{2} \log _{10}(D)+c_{3} D^{c_{4}}+c_{5} D$

(Lehmann \& Häupl 1988), where $I$ designates the intensity of the light the emulsion was exposed to, and $D=-\log _{10}\left(T / T_{\max }\right)$ the resulting photographic density. The coefficients $c_{2}$ to $c_{5}$ are derived for each plate separately from a least-square fit onto the calibration wedge. $c_{1}$ is determined by the aperture photometry procedure described below.

In the next step, the local sky background has to be subtracted. The local sky background is assumed to be 
constant in each sub-frame of (usually) $200^{\prime \prime} \times 200^{\prime \prime}$. Since the galaxy images cover only a small fraction of each frame, the mean local sky background has been assumed to be equal to the well defined maximum of the intensity histogram of the image.

It is well-known that background subtraction is rather difficult when very faint signals of extended objects have to be discriminated against a much brighter sky background, especially in the irregular environment of a galaxy cluster (e.g. Capaccioli \& de Vaucouleurs 1983; Okamura 1988). The photometric errors resulting from our simple background subtraction procedure are expected to become significant for the faintest detectable features. However, it is not our intention to achieve highly accurate photometry of very faint structures, although their visual inspection is very important in the context of morphological classification (see Sect. 5). The apparent magnitudes are derived from the integrated brightness of the galaxies within the $\mu_{B}=25 \mathrm{mag} \operatorname{arcsec}^{-2}$ isophote. The latter corresponds to $5 \%$ of the sky background level which is well above the noise level.

Prior to the determination of isophotal parameters, plate faults as well as foreground stars and overlapping galaxies have to be eliminated carefully. The availability of five co-centred images of each galaxy simplifies the removal of spurious objects. At each pixel position, both the highest and the lowest measured intensity values are omitted. Only the three medium intensity values are averaged to construct a final, "clean" image for each galaxy.

Due to the low Galactic latitude the investigated field is strongly contaminated by foreground stars. Since the approximation of stellar images by a Gaussian distribution and subsequent removal does not always lead to satisfying results, we have decided to remove disturbing object images manually. Stellar images projected on the outer parts of the galaxy images are either removed by the subtraction of the fitted Gaussian profiles, by simply replacing the affected pixel values by those from similar unaffected parts of the image, or by means of the MIDAS procedure MODIFY/AREA. The removal of disturbing images near the galaxy centre is more complicated and time-consuming; in the case of $\mathrm{E}$ or S0 galaxies good results are achieved by first determining the galaxy centre and then replacing the affected pixel values by those on exactly the opposite side of the image at the same distance from the centre. In a few cases, when none of the methods mentioned above worked properly, we have used a pixel editor to reconstruct the undisturbed image as well as possible. Throughout the cleaning process, the images have been monitored on the display in order to avoid any misfits. Although manual star removal is time-consuming and seems to be somewhat inaccurate, it rather well preserves galaxy features that may be removed by automated cleaning procedures.

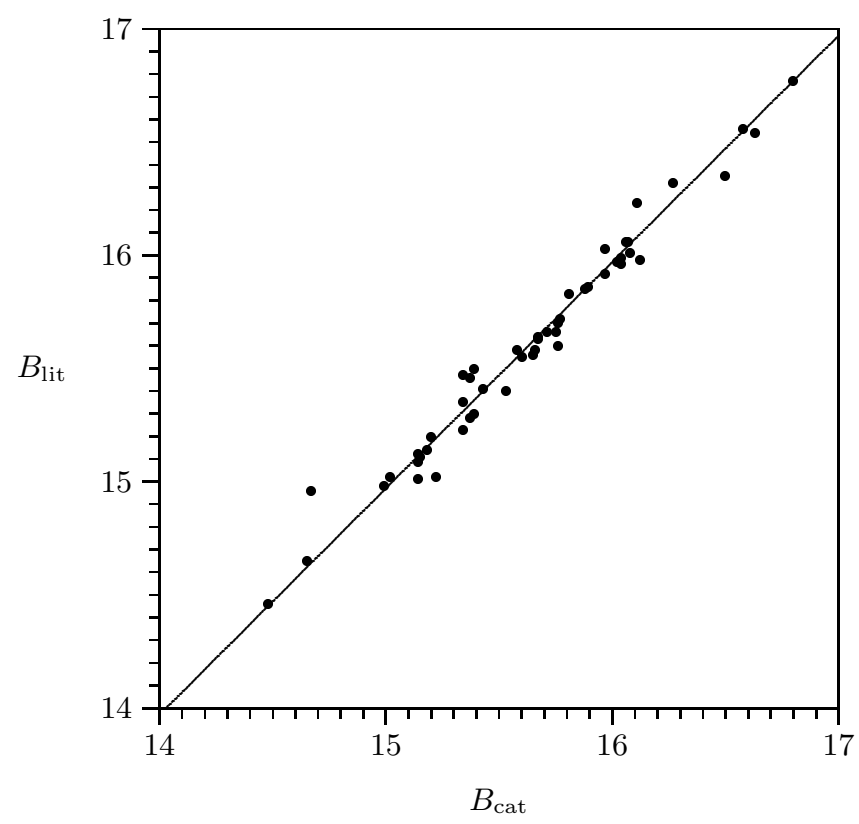

Fig. 2. Photometric zero point calibration: aperture photometry of galaxies of the present catalogue $\left(B_{\text {cat }}\right)$, compared with results published by Weedman (1975) and Strom \& Strom $(1978)\left(B_{\text {lit }}\right)$. Mean standard deviation $\sigma\left(B_{\text {lit }}-B_{\text {cat }}\right)=$ $0.08 \mathrm{mag}$

For the absolute flux calibration of the images, the results of the photoelectric and photographic aperture photometry of A 426 galaxies by Weedman (1975) and Strom \& Strom (1978) serve as reference. We have only considered apertures $\geq 17^{\prime \prime}$ to avoid seeing and sampling effects. NGC 1275 has been excluded from the calibration sample mainly due to a close foreground star. For the remaining 50 galaxies, the internal nuclear magnitudes have been determined from numerical integration of the relative intensities within the corresponding aperture. After subtraction of the mean offset between the published and the measured nuclear aperture magnitudes, the remaining rms difference is $0.08 \mathrm{mag}$ (Fig. 2). The systematic error of the absolute calibration calculates as $0.08 \mathrm{mag} / \sqrt{50}=0.01 \mathrm{mag}$.

From the numerical integration of the calibrated intensities within the $\mu_{B}=25 \mathrm{mag}_{\operatorname{arcsec}}{ }^{-2}$ isophote, the apparent magnitude $B_{25}$ of each galaxy has been derived. The total error is estimated to be of the order of 0.1. . 0.2 mag. No colour correction has been applied since the Tautenburg $B$ band almost exactly matches the Johnson $B$ band for $0<(B-V)<2$ (van den Bergh 1964; Börngen \& Chatchikjan 1967; Andruk et al. 1994). Major and minor axis as well as position angles (PA) of the galaxies have been also derived from the $25 \mathrm{mag} \mathrm{arcsec}^{-2}$ isophotal ellipse and are included in the catalogue.

The sky background is $\mu_{B}=21.7 \pm 0.1 \mathrm{mag} \mathrm{arcsec}^{-2}$ for all five plates. Surface brightness fluctuations introduced by the granularity are of the order of $0.07 \mathrm{mag} \mathrm{arcsec}^{-2}$ at the $10 \mu \mathrm{m}$ scale for surface brightnesses up to 19 mag $\operatorname{arcsec}^{-2}$. 


\subsection{Radial velocities}

We have derived radial velocities for five of the catalogued galaxies from low-dispersion spectra taken at Calar Alto (Sect. 2). The radial velocities of two IRAS galaxies could easily be measured thanks to the presence of prominent emission lines in their spectra. No strong emission lines have been found for the other galaxies, but absorption features of a late type stellar population were unambiguously identified: Ca II K\&H $\lambda \lambda 3933,3968, G$ band $\lambda 4307$, Mg I $\lambda 5175, \mathrm{Na}$ I $\lambda 5893$. The resulting heliocentric radial velocities are included in the catalogue. The typical uncertainties are $\sim 200 \mathrm{~km} \mathrm{~s}^{-1}$.

\section{Morphology}

\subsection{Procedure of evaluating morphology}

Morphological properties of the galaxies have been deduced from the visual inspection of (a) deep images obtained from digital co-addition of the images from all measured plates, (b) digitised images obtained from single high quality plates taken under good seeing conditions, (c) radial intensity profiles from surface photometry, and (d) the radial run of the position angle.

Co-addition of Schmidt-plates is known to be a powerful method for the evaluation of faint features in galaxies (e.g. Malin \& Carter 1993; Schweizer \& Seitzer 1988; Malin 1994; Kemp \& Meaburn 1995). Up to 37 images have been digitally centred and co-added for every galaxy. Due to the significantly increased signal-tonoise ratio, faint features down to a surface brightness of $27 \mathrm{mag} \mathrm{arcsec}^{-2}$ can be recognized. On the other hand, the bright inner parts of galaxies as well as any features affected by bright foreground stars are not well studied on the stacked images, primarily owing to the rather poor seeing conditions for the majority of the plates. Therefore, a combination of the deep, co-added image of a certain galaxy with its image on a single high-quality plate (seeing $<1^{\prime \prime}$ ) is the most appropriate way to analyse the information on structure details.

Three independent inspection runs were performed including all 660 galaxies in every run. For each galaxy a page of six different pictures was inspected containing the direct images from the co-added plates and the one from the best single plate, respectively, each at two different magnifications and combined with the corresponding contour plots from these both images. Artifacts like plate flaws or artificial Earth satellites are, in general, strongly suppressed in the co-added image due to plate stacking. With regard to very faint structure details in the galaxy images however, one has to ensure that unusual features detected are real. In these cases, all single images involved in the co-added image had to be inspected. The whole procedure of evaluating morphology was rather time-consuming but proves to provide quite reliable results.

\subsection{Morphological type classification}

One of the main aims of the study of morphology is to derive estimates of the morphological types. It should be emphasized that the galaxy images have been observed in the $B$ band where most existing schemes for morphological classification of galaxies have been defined. Unfortunately, only a rough discrimination between E/S0, S and Irr types is possible for most galaxies on Schmidt plates at the distance of the Perseus cluster. (The notation Irr is used as well for normal disk-dominated late-type galaxies Sdm, $\mathrm{Sm}, \mathrm{Im}$ as for galaxies which cannot be assigned Hubbletypes.)

The presence of an apparent spiral structure is taken as the main criterion for the classification of spiral galaxies. In this context, the use of blue-sensitive plates is important to ensure the detection of features related to recent star formation, like spiral arms. We searched for indications of spiral arms on (a) the direct images, (b) the contour plots, and in (c) the radial run of the position angles. Unfortunately, the appearance of spiral structure can be mimicked by triaxiality in early type galaxies or even by a superposition of faint foreground stars. In addition, radial luminosity profiles have been analysed for 79 sufficiently extended galaxies. For most galaxies the types estimated from these different methods were in good agreement.

For many galaxies of the sample it is difficult to decide clearly between E, S0 and S types. In these cases, two types are given in the catalogue with the more likely one at first position. If an alternative type is less likely, but cannot be excluded, it is quoted with a question-mark. Classification has been completely impossible for less than $10 \%$ of the galaxies. This concerns small and faint objects, often located close to bright foreground stars. For spiral galaxies it is sometimes possible to dicriminate between early- and late-type, mainly from the bulge-to-disk ratio.

For the galaxies with isophotal analysis by Poulin et al. (1992), the agreement with regard to the three basic morphological types (E/S0, S, Irr/Pec) is very good: among the 43 galaxies contained in both lists and unambiguously classified by one single type, there are 39 galaxies (i.e. 91\%) where the estimated types from both lists are in agreement; for two galaxies no classification has been possible in our study. For the 35 galaxies where two possible types are given in at least one of the two lists, 33 classifications (94\%) have at least one type in common. Furthermore, for the 30 galaxies from our catalogue contained in the lists of Perseus galaxies by Andreon et al. (1997a) we find a similar result: among the galaxies classified as early types by Andreon et al. (their Table 5) we have 3 out of 16 objects in our catalogue with differing classification ( $\mathrm{S}$ in our catalogue, $\mathrm{S} 0$ in Andreon et al.). 
For 14 galaxies classified as $\mathrm{S}$ by Andreon et al. (their Table 3) we find a disagreement only for one (No. 120), which we classify as S0. As reliable estimates of morphological types have been the main goal of these both studies, we conclude from this comparison that our classification as $\mathrm{E}+\mathrm{S} 0$, S, and Irr types should be correct for about $90 \%$ of the brighter galaxies $(B<16)$. The uncertainties increase for fainter and/or smaller galaxies.

\subsection{Morphological peculiarities}

Many inspected galaxies show faint morphological peculiarities in the co-added images. Despite different attempts (e.g. Naim et al. 1997), quantifying peculiarity is still a challenge. During a "training phase", we studied the appearance of catalogued peculiar galaxies (Arp \& Madore 1987; Whitmore et al. 1990), of various individual interacting galaxies, and of typical features appearing in numerical simulations of galaxy encounters (e.g. Barnes 1992; Howard et al. 1993). After a first inspection run of our images of the A 426 galaxies, we decided to use a classification scheme for detected peculiar structures which is essentially a combination of the schemes by Karachentsev (1972), Arp \& Madore (1987), and Whitmore et al. (1990) with four basic types:

$I N$ : systems with signs of strong interaction in the form of bridges (br) and/or tails (ta).

$D I S$ : systems with signs of distortions, which can show one or several of the following features coded by a number: peculiar spiral arms (1), three-armed spirals (2), M 51 type (3), unusual dust absorption (4), elliptical galaxy with isophote twist (5), non-concentric core (6), loops/shells (7), jet-like structures (8), fuzzy structure in the outer regions (9), radial spikes (10).

$C H(n): n$ systems $(n>1)$ within a common halo.

$P L$ : polar ring galaxies or related objects (see Whitmore et al. 1990).

\section{Description of the catalogue}

The catalogue of 660 galaxies in a field of about 10 square degrees is available in electronic form at the CDS via anonymous ftp to cdsarc.u-strasbg.fr (130.79.128.5) or via http://cdsweb.u-strasbg.fr/Abstract.html. In this section, we give detailed information on the structure and the content of the catalogue:

Column (1) Running number.

Column (2) Cross-identification, taken from $\mathrm{NED}^{1}$ or PGC (Paturel et al. 1989, 1993). Only the most common

\footnotetext{
1 The NASA/IPAC Extragalactic Database (NED) is operated by the Jet Propulsion Laboratory, California Institute of Technology, under contract with the National Aeronautics and Space Administration.
}

name is given, i.e. NGC, IC, UGC, PGC number, or NED object name (in this order). The cross-identification is primarily based on positions. For positional differences $<4^{\prime \prime}$ we assume that the corresponding galaxies are identical. For differences from $4^{\prime \prime}$ to $60^{\prime \prime}$ cross-identifications are given only if (a) the positions agree within the given error boxes and (b) any misidentification can be ruled out. A few UGC galaxies with larger positional errors could be identified as well.

Column (3) Right ascension $\alpha$ for equinox 2000, in hours, minutes, and seconds. The mean error is $\sim 0$ s.03.

Column (4) Declination $\delta$ for equinox 2000, in degrees, arcminutes, and arcseconds. The mean error is 0.3 .

Column (5) Apparent magnitude $B_{25}$ within a limiting isophote of $\mu_{B}=25 \mathrm{mag} \operatorname{arcsec}^{-2}$. No corrections regarding Galactic extinction, inclination and $K$-dimming have been applied. Due to image crowding by galaxies and foreground stars the mean uncertainty is about 0.1 to $0.2 \mathrm{mag}$. Larger uncertainties due to difficulties in the removal of foreground stars are marked by a colon.

Column (6) Central $B$-surface brightness $\mu_{\mathrm{C}}$ determined from the central $5 \operatorname{arcsec}^{2}$ of the galaxy, in mag $\operatorname{arcsec}^{-2}$.

Column (7) Major axis $a$ of an ellipse fitted on the $\mu_{B}=25 \mathrm{mag} \mathrm{arcsec}^{-2}$ isophote, in arcsec. The mean error is less than $10 \%$.

Column (8) Minor axis $b$ of an ellipse fitted on the $\mu_{B}=25 \mathrm{mag}_{\operatorname{arcsec}^{-2}}$ isophote, in arcsec. The mean error is less than $10 \%$.

Column (9) Position angle PA, in degrees East of North. The accuracy depends on the ellipticity; usually the given PAs agree within a few degrees with the PGC data.

Column (10) Heliocentric radial velocity $v_{\text {rad }}$, in $\mathrm{km} \mathrm{s}^{-1}$. For 166 galaxies, radial velocity data have been available either from the NED or from the PGC, respectively. For the galaxies $329,335,342,353$, and 540, radial velocities have been determined in the present study (see Sect. 4.3).

Column (11) Morphological type class (see Sect. 5).

Column (12) Remarks. If the estimation of the morphological type is hampered by a nearby foreground star this is indicated by "f $*$ ". Morphological peculiarities are coded as DIS, IN, CH, PL (Sect. 5.3) with strength indicated by "+" (strong) or "_" (weak). If other galaxies from the catalogue are related to the mentioned peculiarity, their catalogue numbers are given in brackets. 


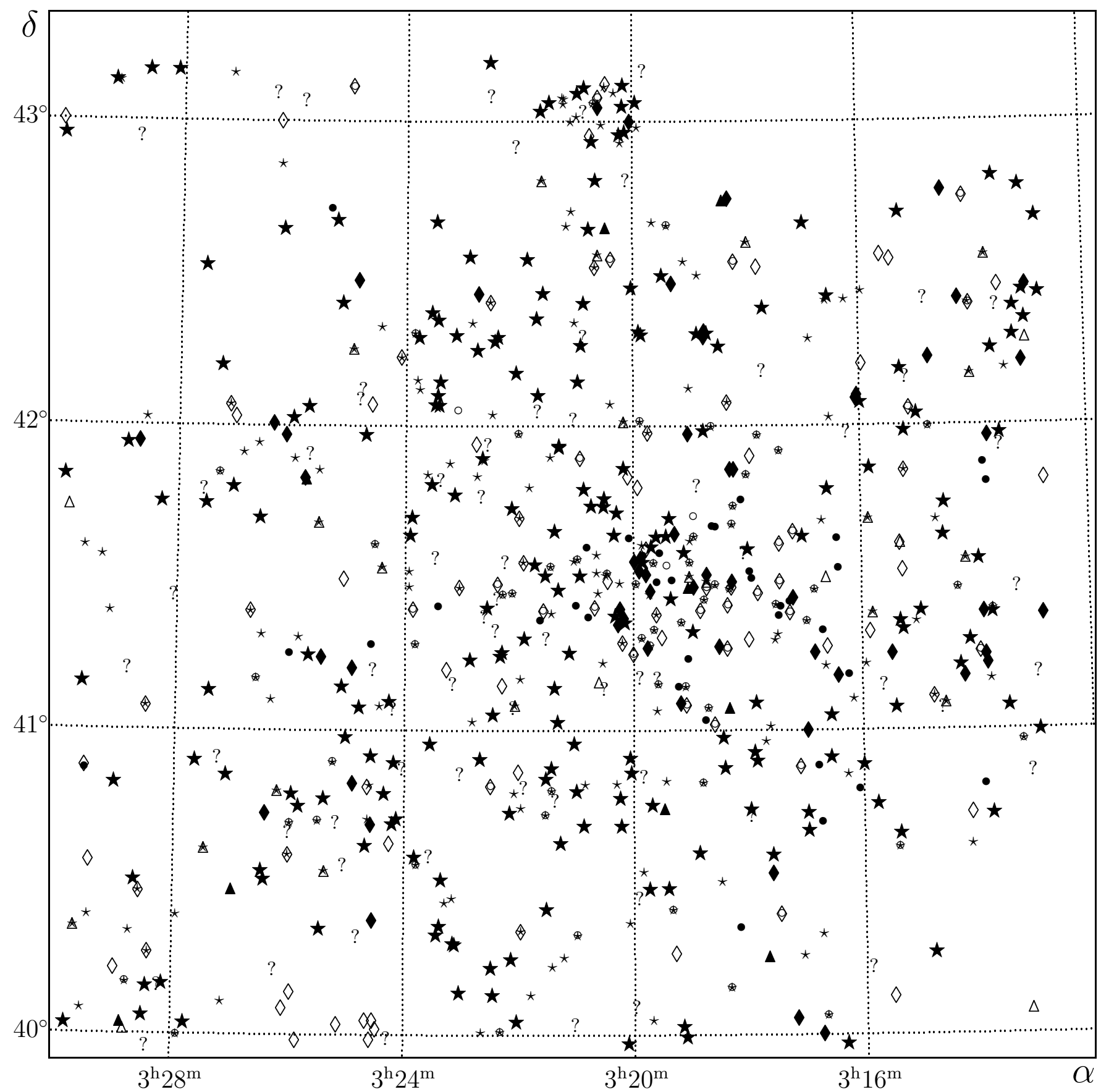

Fig. 3. Distribution of catalogued galaxies on a Tautenburg Schmidt plate centred on $\alpha=3^{\mathrm{h}} 21^{\mathrm{m}} 10^{\mathrm{s}}$ and $\delta=41^{\circ} 33^{\prime}(\mathrm{J} 2000)$. The gap in the upper right corner is due the calibration wedge. The size of the symbols indicates the brightness of the galaxies. $(\mathrm{S}=\star, \mathrm{S} ?=\star, \operatorname{Irr}=\boldsymbol{\Delta}, \operatorname{Irr} ?=\Delta, \mathrm{E}=\star, \mathrm{E} ?=\diamond, \mathrm{S} 0=\bullet, \mathrm{S} 0 ?=0$, unknown=?)

7. Statistical properties of the galaxies in the Perseus cluster

\subsection{Distribution of the galaxies in the field}

The distribution of all 660 catalogued galaxies is shown in Fig. 3. Note that the northwest corner of the field is reserved for the calibration wedge, hence no objects are recorded there. The lack of galaxies to the northeast of the cluster centre has been already reported by KS 83 and was explained there as due to a sharp increase in the foreground extinction. A second lack is indicated toward the southwest of the cluster centre. Indeed, the IRAS $100 \mu \mathrm{m}$ map (see Ettori et al. 1998, their Fig. 7) clearly shows enhanced absorption in both of these regions. Because of the low Galactic latitude of the field, the foreground extinction is expected to be rather irregular and may pretend 


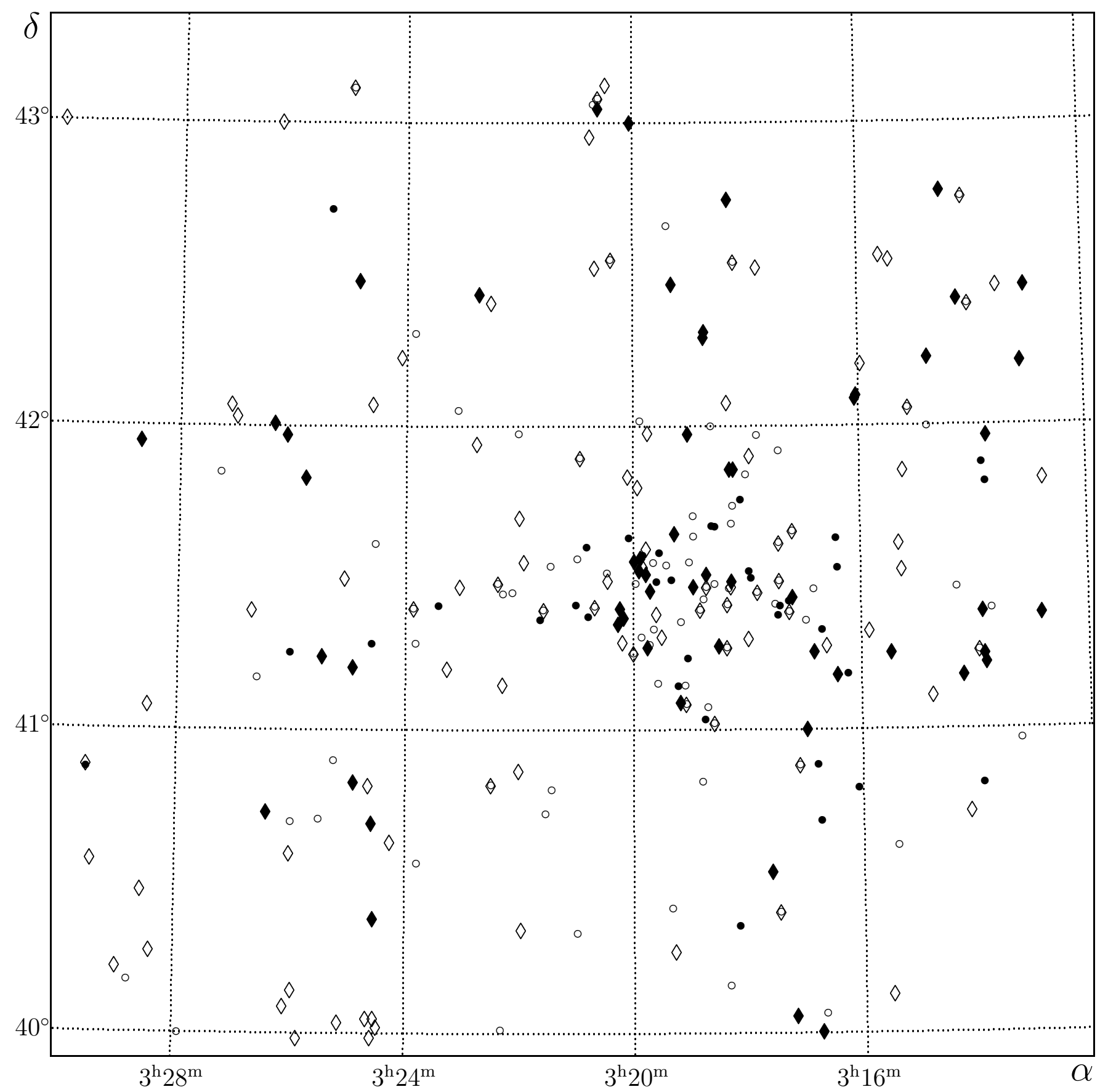

Fig. 4. As Fig. 3, but only for $\mathrm{S} 0$ and elliptical galaxies. $(\mathrm{E}=\bullet, \mathrm{E} ?=\diamond, \mathrm{S} 0=\bullet, \mathrm{S} 0 ?=\circ)$

substructures in the cluster. Therefore, we are not inclined to study details in the projected galaxy distribution.

However, we have to explain the prominent clump of galaxies at $\alpha(\mathrm{J} 2000.0)=3^{\mathrm{h}} 20 .{ }^{\mathrm{m}} 4, \delta(\mathrm{J} 2000.0)=43^{\circ} 4^{\prime}$, (about 1.5 north of the Perseus cluster centre), clearly seen in Fig. 3. This structure has not been reported before; it is not obvious from the Zwicky sample discussed by KS 83 and Andreon (1994). The strong concentration and the faint magnitudes of the galaxies of the clump (typically $B>17$ ) point towards a background cluster. The bright- est member (no. 335) has $B_{25}=16.1$ and is classified as $\mathrm{E} / \mathrm{S} 0$ ? with several faint nearby objects within a common halo. It is identified with the radio source B3 $0317+428$. From the spectra obtained for this galaxy and two other members of the clump (no. 329 and 353), a redshift of $z=0.050 \pm 0.001$ has been derived (Sect. 4.3), confirming that it is indeed a background cluster. Nevertheless, we do not explicitly exclude this cluster from our sample in the further analysis, because background contamination will be corrected statistically. 


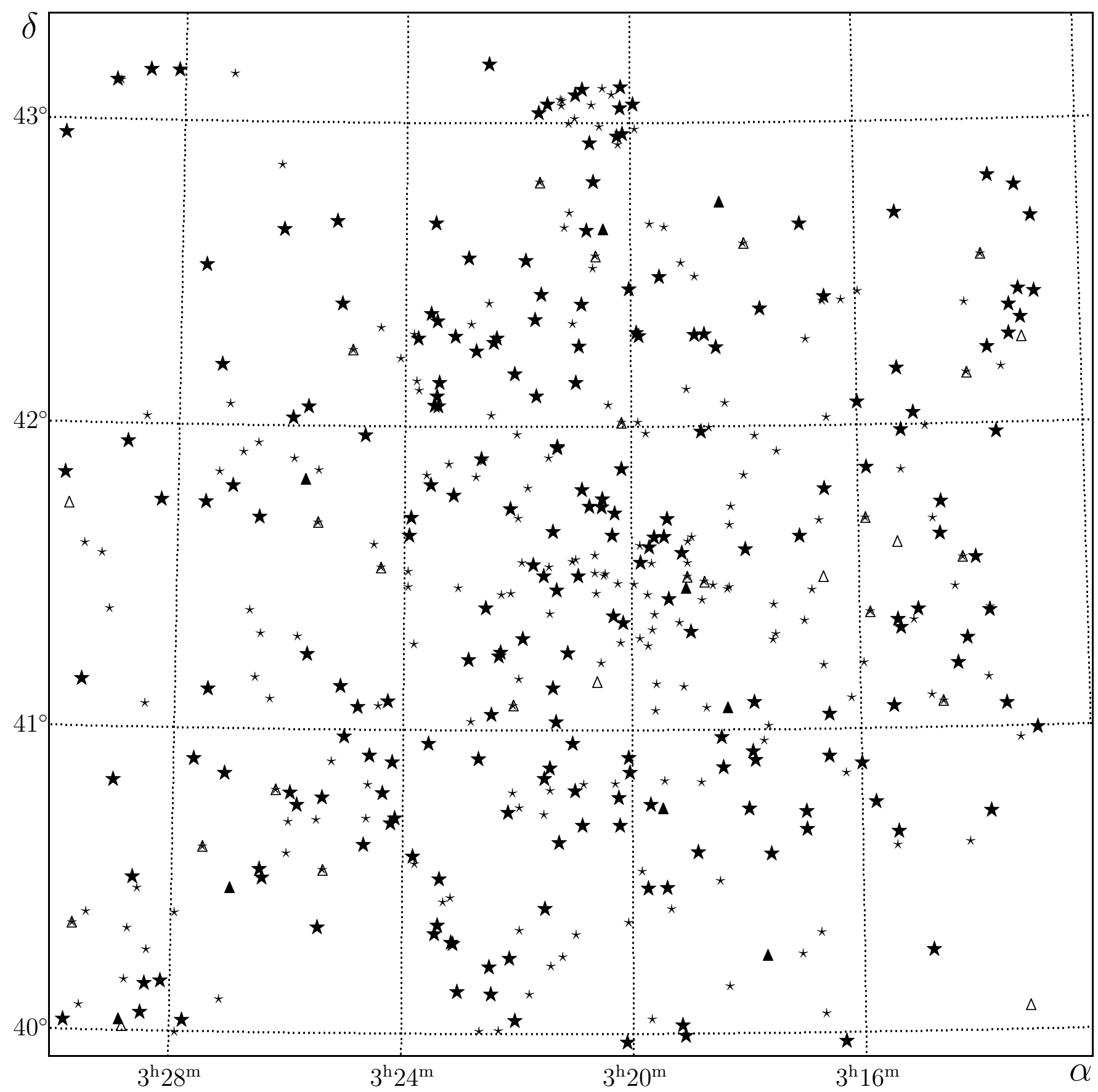

Fig. 5. As Fig. 3, but only for spiral and irregular galaxies. $(S=\star, S ?=\star$, Irr $=\boldsymbol{\Delta}, \operatorname{Irr} ?=\Delta)$

As is well known, the bright galaxies in the centre of A 426 are aligned along a prominent chain. Such a chain is not clearly seen in the distribution of all catalogued galaxies, and a flattening of the cluster is only marginally indicated. This has been noticed already by KS 83 .

\subsection{Background galaxies}

Contamination by background galaxies is difficult to quantify. From the distribution of radial velocities for 187 galaxies in A 426, KS 83 estimated maximum radial velocities for cluster members in dependence on the distance $r$ from the centre. We adopt this relation to discriminate between members and non-members. Unfortunately, radial velocities are available only for about $25 \%$ of the catalogued galaxies. Under the assumption that this subsample is not strongly biased towards cluster members we expect about 3, 10, and 27 background galaxies for limiting magnitudes $B_{\text {lim }}=16,17$, and 18 , respectively. This, however, is presumably an underestimation of the true 
background contamination, since most galaxies have been measured in the inner region $\left(r<30^{\prime}\right)$, where both the fraction of cluster galaxies and the velocity dispersion are very large. KS 83 argued that nearly all of the galaxies to a limiting magnitude $V=17.5$ in the central region belong to the cluster.

For an independent estimate of the number of background galaxies we use the number-magnitude relation for field galaxies quoted by Binggeli et al. (1988) along with an adopted mean extinction of $A_{B}=0.8$ toward A 426 (KS 83):

$\log _{10} N_{B}(B)=0.6 B-9.79$,

where $N_{B}(B)$ designates the number of background galaxies brighter than $B$ per square degree. For galaxies with a mean colour index $(B-V)_{0} \approx 0.7$ this relation is in agreement with the galaxy background densities adopted for A 426 by $\mathrm{KS} 83$. As emphasised by KS 83, these estimates have to be regarded as quite uncertain.

From the comparison of Eq. (2) with the luminosity function of all galaxies (Fig. 12), we conclude that background contamination should be negligible for $B_{25}<17$, but becomes significant for $B_{25}>18$. This seems to be indicated also by the radial profile of the galaxy number density: if the number density of background galaxies was significantly underestimated by Eq. (2), the backgroundcorrected profiles are expected to become shallower at large $r$. This is, however, not observed, at least for galaxies with $B_{25}<17$ (Fig. 7).

For the following statistical investigations we will, therefore, consider either the subsample of galaxies with $B_{25}<17.5$ or both the galaxies with $B_{25}<17$ and those with $B_{25}<18$ for comparison.

\subsection{The optical centre of the Perseus cluster}

A 426 belongs to the clusters presenting an offset between the optical centre and the X-ray centre. Such offsets may be the signature of a recent merger (Ulmer et al. 1992). Moreover, the choice of the cluster centre is crucial to the determination of the central density profile (Beers \& Tonry 1986; see also next subsection). In the context of the present work, a complete discussion of the topic can not be presented, we refer the reader to Ulmer et al. (1992), Casertano \& Hut (1985) and Beers \& Tonry (1986).

Following Ulmer et al. (1992), we determine both the density centre and the luminosity centre. In both cases, increased weight is given to the (mainly brighter) galaxies in the core, and reduced weight to isolated (mainly fainter) outliers, especially randomly distributed background galaxies. The estimates are, however, to a certain degree sensitive to substructures in the projected galaxy distribution.

The density centre is derived from the densityweighted mean of all galaxy positions within a circular area of radius $r$ centred on NGC 1275. This reference

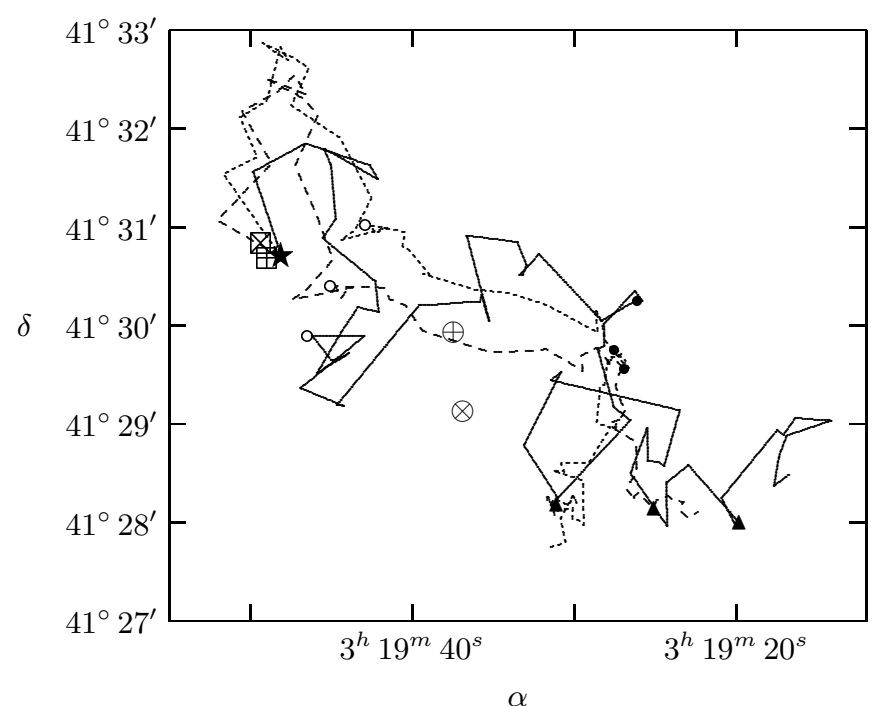

Fig. 6. Wandering of the deduced optical centre of the Perseus cluster. Shown is the traced position of the cluster centre as derived from the intensity- or density-weighted positions of the galaxies within a certain distance $r$ from NGC 1275 (marked by $\star$ ). The distance $r$ varies from $1^{\prime}$ to $70^{\prime}$ in steps of $1^{\prime}$. Solid line: trace of the luminosity centre of all galaxies, dashed lines: trace of the density centre of all galaxies with $B_{25} \leq 18$ (short dashes), and $B_{25} \leq 17$ (long dashes), respectively, both corrected for a constant background galaxy contamination. The positions of the centres at $r=20^{\prime}, 40^{\prime}$, and $60^{\prime}$ are marked by small open circles, filled circles, and triangles, respectively. Cluster centre positions given by Ulmer et al. (1992) are also marked $(\oplus$ : median centre of optical galaxies, $\otimes$ : density centre

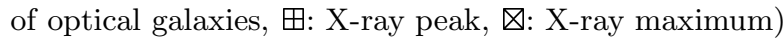

point has been used since (a) it is obviously located near the centre of the dense central region (see Fig. 3 ; cf. also KS 83), and (b) its position almost exactly coincidences with the peak of the X-ray emission of the cluster (Ulmer et al. 1992). Each galaxy position is weighted by its inverse projected distance to its $N$ th nearest neighbour, which is a measure of the local density of galaxies. We set $N=6$ because the weights do not significantly change with $N$ for $N \geq 5$. To reduce the influence of background galaxies, all galaxies fainter than 18 have been excluded from the calculation. We have repeated the calculations considering only the galaxies brighter than 17 . In addition, the weights have been corrected for an assumed constant background galaxy density $N\left(B_{25} \leq 18\right)=10.2 \mathrm{gal} \mathrm{deg}^{-2}$, and $N\left(B_{25} \leq 17\right)=2.57$ gal deg $^{-2}$, respectively (Eq. 2 ). For the calculation of the luminosity centre the $B$ luminosities are used as weights. No corrections for background galaxies have been applied since they are negligible.

We calculate the centre positions for radii $r=1 \ldots 70^{\prime}$ in steps of $1^{\prime}$ and trace the resulting shifts of the centre. The wandering of the differently calculated centres is shown in Fig. 6. 


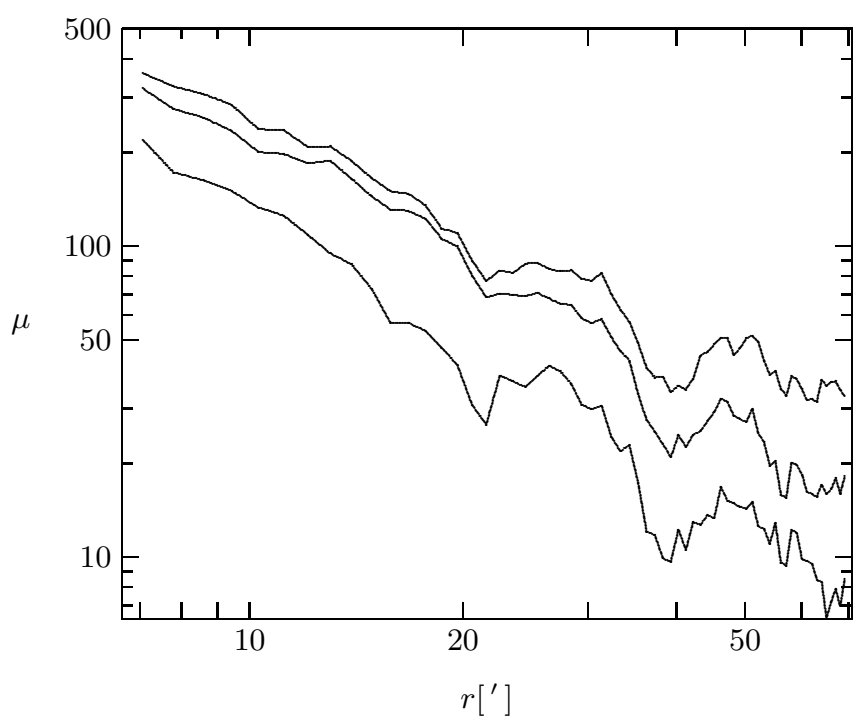

Fig. 7. Surface density profile of the Perseus cluster. The number of galaxies per square degree $\mu$ in a circular bin of $10^{\prime}$ size is shown as a function of the mean radius $r$ of the bin: $r=\sqrt{r_{\min }^{2}+r_{\max }^{2}}$. From top to bottom: galaxies with $B_{25} \leq 18, \leq 17$ and $\leq 16$. All data are background-corrected (Eq. 2)

The drifts of both the density and the luminosity centre are remarkably similar. This seems to indicate that the distributions of the brighter and fainter galaxies are similar, even though not obvious from the inspection of the projected distribution of the galaxies. The calculation of the centre position is only marginally influenced by background galaxies, as shown by the similarity between the density centre tracks for galaxies with $B_{25}<17$ and 18 , respectively. The drifts do not significantly depend on the choice of the reference point. However, it can not be ruled out that they are affected by patchy foreground extinction.

We now calculate the positions of the cluster centres for each galaxy type separately. For radii $r=10^{\prime} \ldots 50^{\prime}$, the cluster centre of spirals is shifted as far as $\sim 8^{\prime}$ east of NGC 1275, whereas E+S0 galaxies cluster rather west of NGC 1275, reflecting the prominent chain which consists mainly of Es and S0s (cf. Figs. 4 and 5). This result is opposite to the morphological centres derived by Andreon (1994).

Apparently, the asymmetric projected distribution of galaxies hampers the determination of a unique cluster centre. In the following sections, we will adopt the median centre proposed by Ulmer et al. (1992) as cluster centre, i.e. $\alpha_{\mathrm{mc}}=3^{\mathrm{h}} 19^{\mathrm{m}} 37^{\mathrm{s}} .0, \delta_{\mathrm{mc}}=41^{\circ} 30^{\prime} 03^{\prime \prime}$ for J2000.

\subsection{Surface density profile}

The determination of the total extent of the Perseus cluster is hampered by patchy extinction and large-scale galaxy clustering. The projected surface density profile, derived from galaxy number counts in our field, traces the

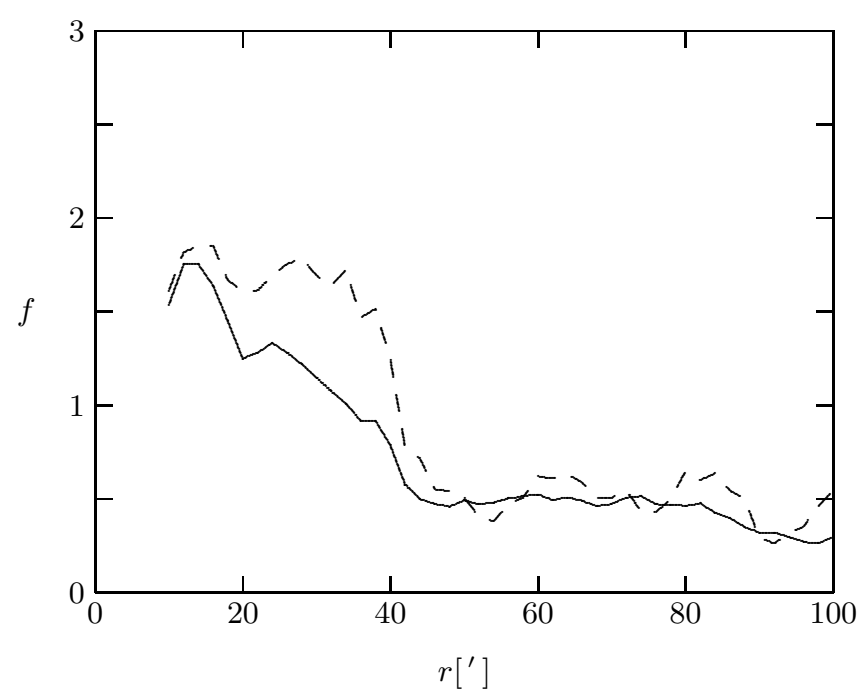

Fig. 8. Radial segregation of morphological types: the number density ratio of early type $(\mathrm{E}+\mathrm{S} 0)$ to late type $(\mathrm{S}+\mathrm{Irr})$ galaxies, $f(r)=N_{\mathrm{E}+\mathrm{SO} 0}(r) / N_{\mathrm{S}+\operatorname{Irr}}(r)$, is computed within $\left[r-15^{\prime}\right.$, $\left.r+15^{\prime}\right]$ centred on $\alpha=3^{\mathrm{h}} 19^{\mathrm{m}} \cdot 7, \delta=41^{\circ} 30^{\prime}$. The solid curve is for all 356 galaxies with $B \leq 17.5$. This sample is complete to a distance $r=65^{\prime}$. The distribution is compared with the sample of 157 galaxies which are expected to be cluster members according to their measured radial velocities (dashed curve)

cluster to radii well beyond $1^{\circ}$ (Fig. 7). We used circular bins with a bin size of $10^{\prime}$, which are centred on the median centre of A 426 proposed by Ulmer et al. (see Sect. 7.3). In three different counts, galaxies with $B_{25} \leq 16, \leq 17$, and $\leq 18$, respectively, have been considered. The obtained galaxy surface densities have been corrected for background contamination according to Eq. (2). Despite some gaps, which might be caused by Galactic extinction and galaxy clustering, the profiles are similar and consistent with a simple power law. The surface density profiles indicate, that the Perseus cluster has a central cusp. This cusp persists if smaller bin sizes (e.g. $5^{\prime}$ ) are applied. This result confirms the finding by Beers \& Tonry (1986) that the presence of central cusps is a general property of rich clusters.

\subsection{Morphological segregation}

A radial morphological segregation in A 426 was already noticed by Melnick \& Sargent (1977), who found that spirals are less concentrated towards the cluster centre than $\mathrm{E}+\mathrm{S} 0$ galaxies. However, their sample contains only $7 \%$ spirals, compared to $50 \%$ in our sample. Andreon (1994) conducted a thorough investigation of the galaxy distribution in the inner region of Perseus, based on a detailed morphological evaluation of the brightest BGP galaxies $(B \leq 15.7)$. He found a strong spatial segregation between the different morphological types and concluded, that the Perseus cluster is dynamically young and not virialized. In the present study, we extend the 

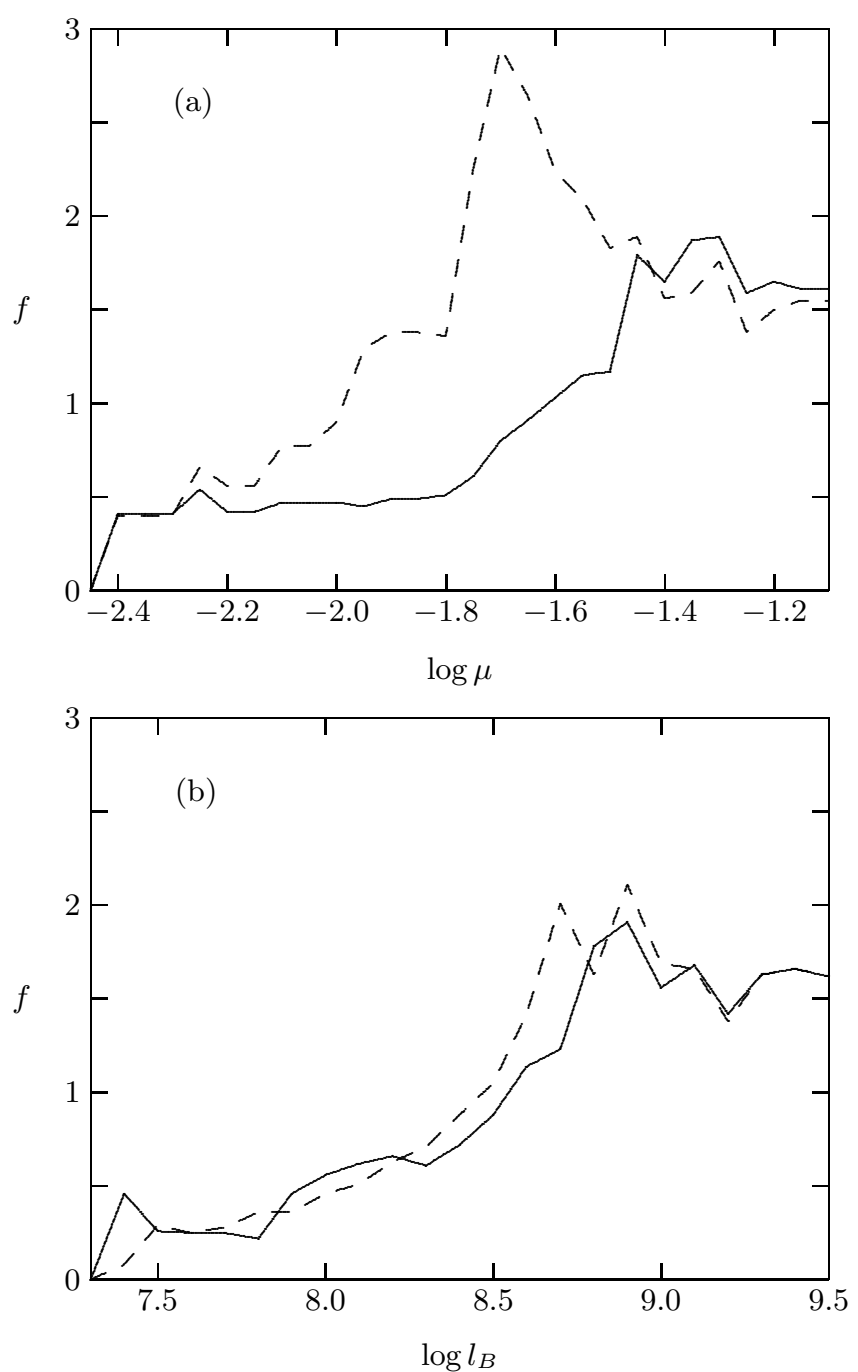

Fig. 9. Density segregation of morphological types: the number density ratio $f$ of early type (E+S0) to late type (S+Irr) galaxies as a function a) of the projected number density $\mu$ (in number per $\square^{\prime}$; top) and $\mathbf{b}$ ) of the $B$ band luminosity density $l_{B}$ (in $L_{\odot, B}$ per $\square^{\prime}$; bottom). The densities are measured within circular areas of $10^{\prime}$ radius; $f(\mu)$ is computed in the intervals $[\log \mu-0.2, \log \mu+0.2], f\left(l_{B}\right)$ in $\left[\log l_{B}-0.2, \log l_{B}+0.2\right]$. Solid curves and dashed curves as in Fig. 8

investigation towards fainter galaxies in a considerably larger field. Preliminary results have been presented by Brunzendorf \& Meusinger (1996).

\section{- Counting convention for morphological types}

Since our morphological classification is ambiguous in many cases (especially for the fainter galaxies), we quantify the accuracy in all following calculations as follows: galaxies classified as type $A$ are counted as $100 \%$ $A$; galaxies of type $A / B$ are considered as $50 \% A$ and $50 \% B$; galaxies of type $A$ ? as $50 \% A$ and $50 \%$ unknown, and finally type $A / B$ ? galaxies as $50 \% A, 25 \% B$ and $25 \%$ unknown. On the basis of this counting convention, we find that $52 \%$ of all 595 classified galaxies are spirals.
If we consider only galaxies with $B_{25} \leq 17$ the spiral fraction is only slightly reduced to $47 \%$.

\section{- Radial segregation}

Whitmore et al. (1993) have argued that the clustercentric distance is the principal determinant of galaxy type within rich clusters. Figures 4 and 5 already indicate that $\mathrm{E}+\mathrm{S} 0$ galaxies dominate the cluster core, whereas S+Irr show only a weak concentration towards the centre. Galaxies of unknown type as well as irregular galaxies show a uniform radial distribution, they contribute $14 \%$ and $3 \%$, respectively, to the sample.

Figure 8 demonstrates the steep drop in the ratio $f$ of early-type $(\mathrm{E}+\mathrm{S} 0)$ to late-type $(\mathrm{S}+\mathrm{Irr})$ galaxies by a factor of more than three. The fraction of identified spirals with $B_{25}=18$ drops from almost $60 \%$ in the outer region down to $30 \%$ in the inner $30^{\prime}$ of the cluster. Thus, the conclusion of a general lack of spirals, reached in previous investigations of the Perseus cluster, turns out to be partly a consequence of the radial morphological segregation in combination with a bias in the galaxy sample. Those studies were based, at least partly, on the BGP sample which covers the central $\sim 30^{\prime}$ only.

\section{- Density segregation}

Dressler (1980) first suggested that morphological segregation is determined by the local galaxy density rather than by the distance from cluster centre. There is a well defined correlation between the fractions of S, S0, and E types and the local projected number density, independent of the cluster concentration. In Fig. 9, we show the "morphological ratio", $f$, for galaxies in A 426 with $B \leq 17.5$ as a function of the projected number density of galaxies and of the projected projected monochromatic $B$ band luminosity density, respectively. A clear tendency is indicated for $\mathrm{E}+\mathrm{S} 0$ galaxies to be relatively more abundant in regions of higher density.

The type-density relation has commonly been related to the active role of substructures in clusters. However, Sanromá \& Salvador-Solé (1990) have shown by means of a very straightforward test that the observed morphological segregation can not be used as an argument in favour of the real existence of clumpiness or substructure.

\section{- Direction segregation}

Andreon (1996, 1998a) and Andreon et al. (1997b) presented arguments in favour of the interesting idea that morphological segregation is primarily based on a privileged cluster direction, perhaps related to the supercluster's main direction. In the clusters studied by Andreon, early-type galaxies show elongated distributions whereas the distribution of spirals is rather isotropic.

In order to study whether a privileged direction is the source of the morphological segregation we discuss the distribution of the galaxies as a function of their clustercentric position angle, $\varphi$. To avoid edge effects, only 
galaxies with distances $r<70^{\prime}$ from the cluster centre are considered. Fig. 10 shows the resulting distributions for $\mathrm{S}+\mathrm{Irr}$ and $\mathrm{E}+\mathrm{S} 0$ galaxies, respectively. Both type classes show a peak at $\varphi \approx 260^{\circ}$ (west), corresponding to the well-known chain of bright galaxies. This peak is stronger for $\mathrm{E}+\mathrm{S} 0$, whereas $\mathrm{S}+\mathrm{Irr}$ show their strongest maximum near $\varphi \approx 40^{\circ}$ (north-east).

For a quantitative analysis of the angular distributions, we apply the Wilcoxon test ( $U$-test), because it is a robust statistical test which requires neither assumptions on the nature of the underlying distribution nor data binning. The $U$-test states whether the distributions of two independent data samples are different by direct comparison of the two sets; it is regarded as one of the most powerful nonparametric tests (e.g., Siegel \& Castellan 1988). To reduce the influence of a possible misclassification, we exclude all galaxies with ambiguous and/or uncertain morphological classification.

Firstly, we compare the cluster-centric galaxy position angles $\varphi$ with those of an artificial sample of the same size but with an isotrope distribution around the cluster centre. The comparison shows that the galaxies are not uniformly distributed around the centre (error probability $\ll 0.1 \%)$, no matter which magnitude range $(B<16$, $B<17, B<18$, all $B$ ) and which galaxy type (S, S0+E, all) is considered. These non-uniformities can be an artifact due to patchy foreground extinction. Therefore, we directly compare the angular distributions of the different morphological types with each other. The probabilities, that each pair of types has the same distribution, are derived from $U$-tests and are listed in Table 2. To maximise the number of galaxies, on the one hand, and to reduce the influence of background galaxies, on the other, we determined the probabilities twice: (a) for galaxies with $B<18$ and (b) for $B<17$. The resulting probabilities are generally similar. We find significant differences (error probability $\sim 0.3 \%$ ) in the angular distribution of E, S0 and E+S0 types compared to S, whereas E and S0 are similarly distributed around the cluster centre. These segregations are detected in the inner part of the cluster $\left(r<30^{\prime}\right)$, which is dominated by E and S0 galaxies, as well as in the outer part $\left(r>30^{\prime}\right)$, where spirals dominate.

Figure 10 suggests a spatial segregation between S+Irr and $\mathrm{E}+\mathrm{S} 0$ galaxies in east-western direction. Therefore, we compare the galaxy distributions of different morphological types in right ascension $\alpha$ and declination $\delta$, again by means of the $U$-test. We consider the inner region $\left(r<30^{\prime}\right)$ and the outer region $\left(30^{\prime}<r<70^{\prime}\right)$ separately. We find statistically significant differences (error probability $\leq 5 \%)$ in $\alpha$ : the probability, that $\mathrm{E}+\mathrm{S} 0$ galaxies and $\mathrm{S}+\operatorname{Irr}$ galaxies with $B<18$ have the same distribution in $\alpha$ is only $0.3 \%$. In contrast, we find no significantly different distributions in $\delta$, even when we choose less stringent significance levels up to error probabilities of $30 \%$ and more. This means that the distributions of $\mathrm{E}+\mathrm{S} 0$ and $\mathrm{S}+$ Irr galaxies indeed differ significantly in right
Table 2. Probabilities (in \%) for each pair of morphological types to have the same angular distribution around the cluster centre. The values refer to galaxies with $B_{25}<18$, values in brackets to galaxies with $B_{25}<17$

\begin{tabular}{|c|c|c|c|}
\hline & $r<30^{\prime}$ & $30^{\prime} \leq r<70^{\prime}$ & $r<70^{\prime}$ \\
\hline $\mathrm{S} / \mathrm{E}$ & $2.4(11.0)$ & $0.5 \quad(5.6)$ & $0.2(11.6)$ \\
\hline $\mathrm{S} / \mathrm{S} 0$ & $2.9 \quad(7.8)$ & $2.6(12.6)$ & $0.5 \quad(5.0)$ \\
\hline $\mathrm{S} 0 / \mathrm{E}$ & $92.8(74.9)$ & $80.3(83.4)$ & $45.3(39.0)$ \\
\hline$(\mathrm{S}+\mathrm{Irr}) /(\mathrm{E}+\mathrm{S} 0)$ & $2.0 \quad(9.5)$ & $0.0 \quad(4.2)$ & $0.3 \quad(9.5)$ \\
\hline
\end{tabular}

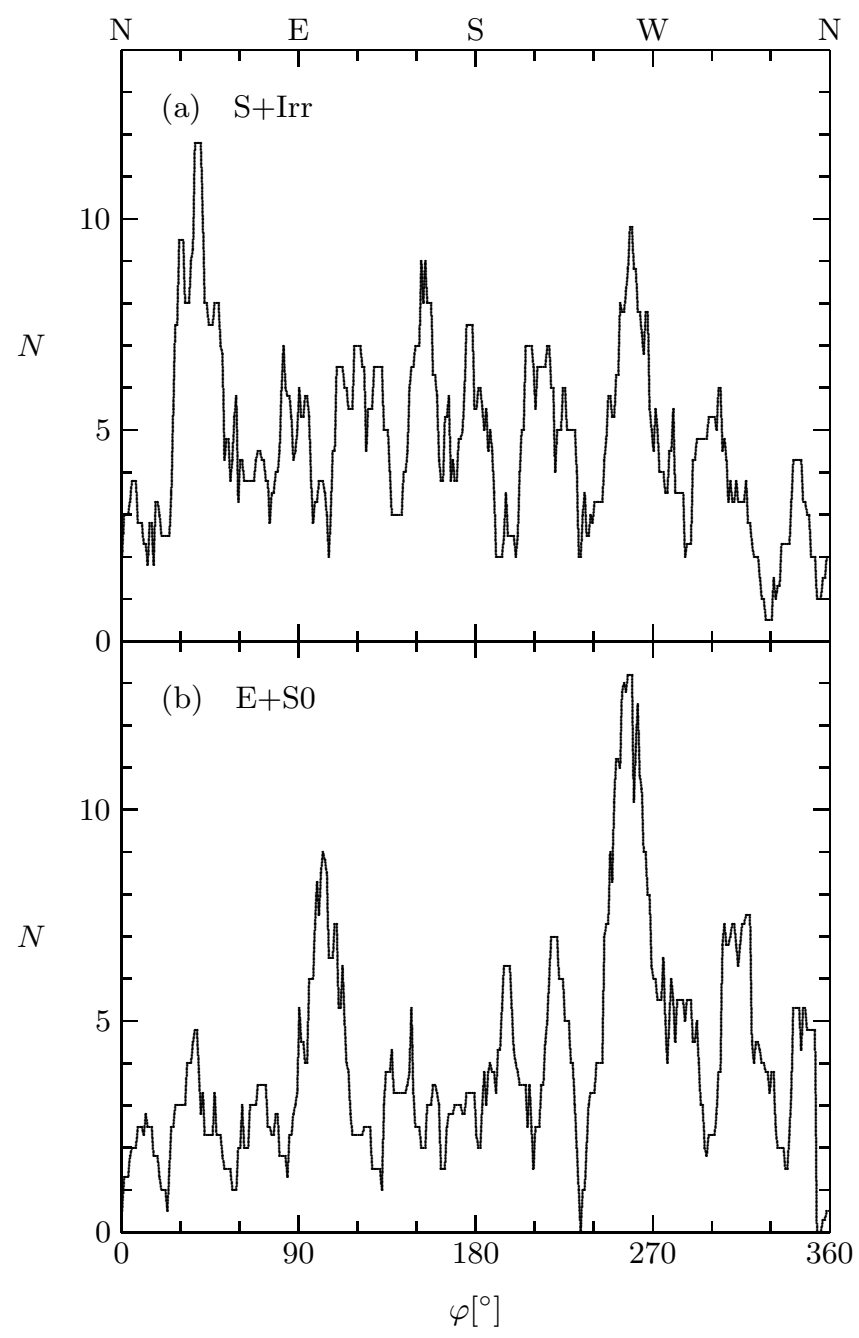

Fig. 10. Angular distribution of galaxies around the cluster centre. The number $N$ of a) $\mathrm{S}+\operatorname{Irr}$ galaxies and $\mathbf{b}) \mathrm{E}+\mathrm{S} 0$ galaxies with a distance $r<70^{\prime}$ from the cluster centre and a polar angle $\varphi$ is binned in intervals of width $\Delta \varphi=15^{\circ}$ 
ascension, whereas a significant deviation in declination could not be found.

A recent analysis of the X-ray surface brightness profile of A 426 from ROSAT PSPC data by Ettori et al. (1998) indicates strong deviations from an isothermal profile both east and west of the X-ray centre. This has been interpreted as evidence for groups merging with the main body of the cluster. While the western deviation has been related to the position of head-tail radio galaxy IC 310, the galaxies in the field of the eastern excess have not been considered yet. In Fig. 11, the "morphological ratio" $f$ is shown for the galaxies in an east-west strip of the width of $\Delta \delta=1^{\circ}$, centred on NGC 1275 (which is very close to the X-ray centre, see e.g. Table 2 in Ettori et al.). Also shown is the distribution of the corresponding number density of galaxies along the strip. The distribution of the brightest galaxies $\left(B_{25} \leq 17\right)$ shows a clear asymmetry, with more galaxies on the western side (the prominent chain). For fainter galaxies $\left(17 \leq B_{25} \leq 18\right)$, the asymmetry is less pronounced with a local maximum at the position of the eastern X-ray excess (between 20 and 50 arcmin from the X-ray centre). Compared with the galaxies in the area of the prominent chain, the morphological mix in the area of the eastern X-ray excess is strongly weighted towards early types. Due to the strong eastward decrease in the number density of bright galaxies there are, unfortunately, only few radial velocities available in this field.

\section{- Summary}

To summarise, we find clear evidence for morphological segregation in the Perseus cluster. A strong radial segregation and a strong density segregation is clearly indicated. A segregation due to a privileged direction is confirmed in several independent tests: the cluster centre of spirals is displaced $\sim 10^{\prime}$ east of the cluster centre of E+S0 galaxies. Furthermore, the angular distribution of both types around the cluster centre and their distribution in right ascension are significantly different. Most pronounced is the privileged eastward direction in the distribution of $\mathrm{E}+\mathrm{S} 0$ types. The analysis of ROSAT PSPC observations of the Perseus cluster yields a clear eastward excess of the distribution of the intracluster medium (Ettori et al. 1998). This fact is in agreement with the conclusion by Andreon et al. (1996) that the privileged direction of morphological segregation in the Perseus cluster is aligned with the direction of the outer isophotes of the X-ray emission.

\subsection{Orientation of galaxies}

The investigation of galaxy alignments may provide clues to the formation and evolution of galaxies, and a large number of studies have searched for non-random effects in the orientation of galaxies relative to larger structures (e.g., Djorgovski 1987, and references therein). The results

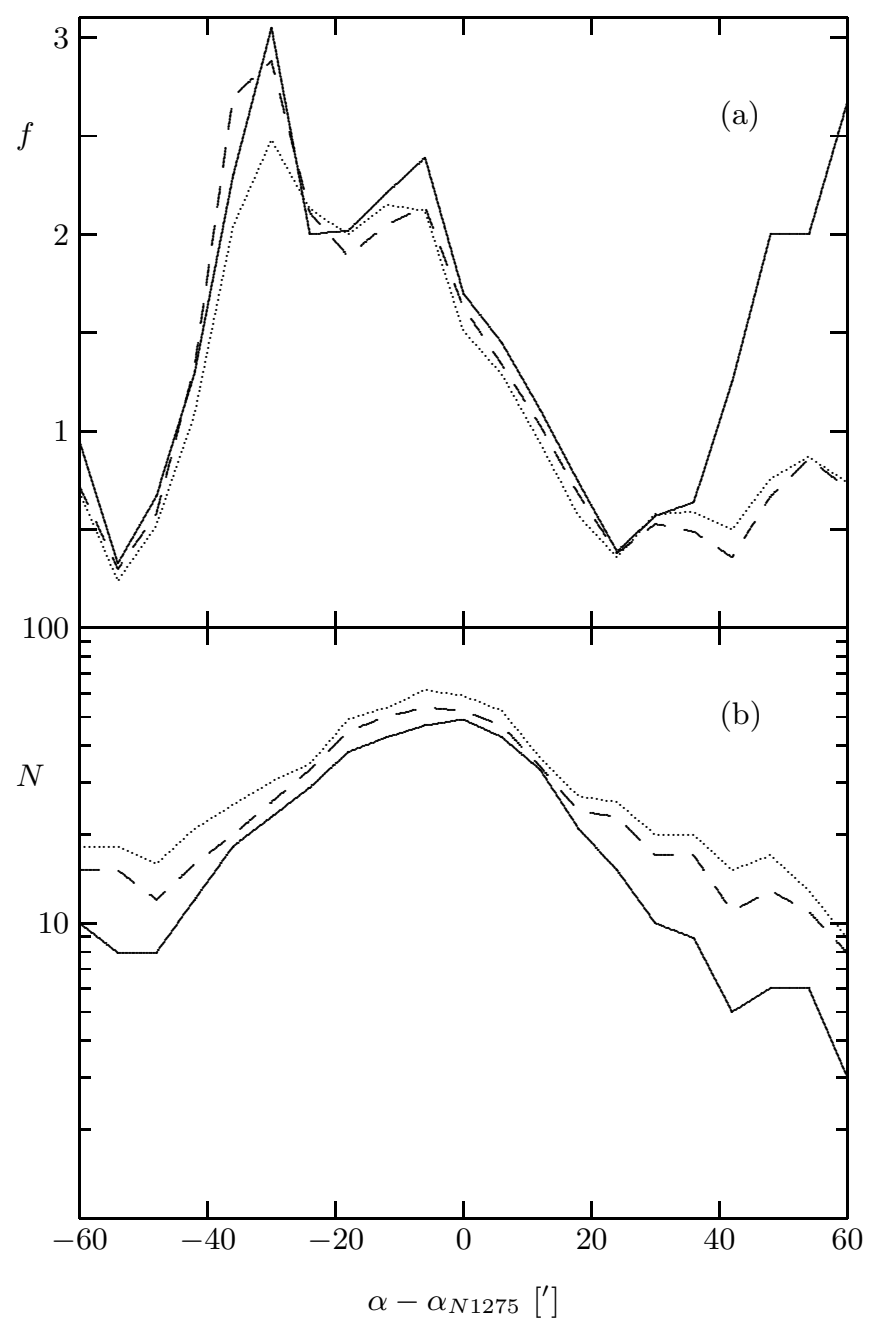

Fig. 11. Morphological segregation in right ascension for galaxies in the central strip of the width of $\Delta \delta=1^{\circ}$, centred on NGC 1275: a) the number density ratio $f$ of early-type (E+S0) to late-type (S+Irr) galaxies and $\mathbf{b}$ ) the number $N$ of galaxies in the $\alpha$ bins used to compute $f$; both $f$ and $N$ were calculated in bins of the width $\Delta \alpha=15^{\prime}$ for galaxies with $B_{25} \leq 17.0$ (solid curve), $\leq 17.5$ (dashed), and $\leq 18.0$ (dotted), respectively

are still rather contradictory, but there are some indications for alignments of disk galaxies in both the Virgo (Hu et al. 1995) and the Coma cluster (Wu et al. 1997) of galaxies. Gregory et al. (1981) found a statistically significant (1.6\% error probability) bimodal distribution for the position angles, PA, of both $\mathrm{E}$ and $\mathrm{S}$ galaxies in the Perseus supercluster, with one peak coinciding with the position angle of the supercluster filament. No correlation of PA with redshift or position in the supercluster was found.

In this subsection, we examine the distribution of PA for the 643 galaxies in the Perseus cluster with an ellipticity $\epsilon \geq 0.3$. Our sample contains considerably more galaxies than the sample investigated by Gregory et al., yet it covers only a small fraction of the Perseus Supercluster. Two different statistical tests are applied to estimate the 
Table 3. Probabilities (in \%) for the position angles PA of spirals $\mathrm{S}, \mathrm{E}+\mathrm{S} 0$ galaxies, and all galaxies, respectively, to be uniformly distributed (Cols. 2 to 4). Last column: probability that $\mathrm{S}+\mathrm{Irr}$ and $\mathrm{E}+\mathrm{S} 0$ galaxies have the same distribution of PA Only galaxies with $r<70^{\prime}$ and ellipticities $\epsilon \geq 0.3$ are considered. We find no evidence for a non-uniform distribution of the position angles

\begin{tabular}{lcccc}
\hline & $\mathrm{S}$ & $\mathrm{S} 0+\mathrm{E}$ & all & $(\mathrm{S}+\mathrm{Irr}) /(\mathrm{E}+\mathrm{S} 0)$ \\
\hline$B_{25}<16$ & 98 & 79 & 50 & 97 \\
$B_{25}<17$ & 58 & 90 & 96 & 81 \\
$B_{25}<18$ & 75 & 95 & 94 & 94 \\
all $B_{25}$ & 79 & 95 & 98 & 85 \\
\hline
\end{tabular}

confidence of the hypothesis that the position angles in our sample are not uniformly distributed. We choose a rather low significance level of $90 \%$ (i.e. error probability 10\%) at which the null hypothesis of a uniform PA distribution is considered to be disproved.

Firstly, we repeat the $\chi^{2}$ tests employed by Gregory et al. (1981) to detect non-uniformities in the PA distribution of (a) galaxies brighter $B_{25}=17$, (b) galaxies brighter $B_{25}=18$, and (c) known cluster members.

The resulting $\chi^{2}$ values either prove that the PA distribution is significantly different from a random distribution, or show that it is consistent with a random one, depending on the chosen starting angle of the first bin. Obviously, the way of binning strongly influences the results. We, therefore, reduce the original bin width of $15^{\circ}$ down to $10^{\circ}, 5^{\circ}, 3^{\circ}, 2^{\circ}$, and $1^{\circ}$, respectively. Still, we can not unambiguously disprove the null hypothesis. The same results are obtained if only one morphological type is considered.

In a second test series, we employ the $U$-test to compare the galaxy position angles with artificial samples of the same size containing uniformly distributed angles. The $U$-test is more powerful than the $\chi^{2}$-test, because it avoids binning. The results are listed in Table 3. For all morphological types, we find no significant difference between the observed distribution of galaxy position angles and a uniform distribution. The PA distribution of the known cluster members is also consistent with a uniform distribution. The PA distribution of E+S0 galaxies and S+Irr galaxies closely match as well.

Finally, we applied the Kolmogorov-Smirnov test to the samples (a) to (c). Again, the results show that the distribution of PA is consistent with a random one on a $90 \%$ significance level.

Our results suggest a uniform distribution of the galaxy position angles, i.e. the Perseus galaxies are not preferably orientated towards a particular direction.

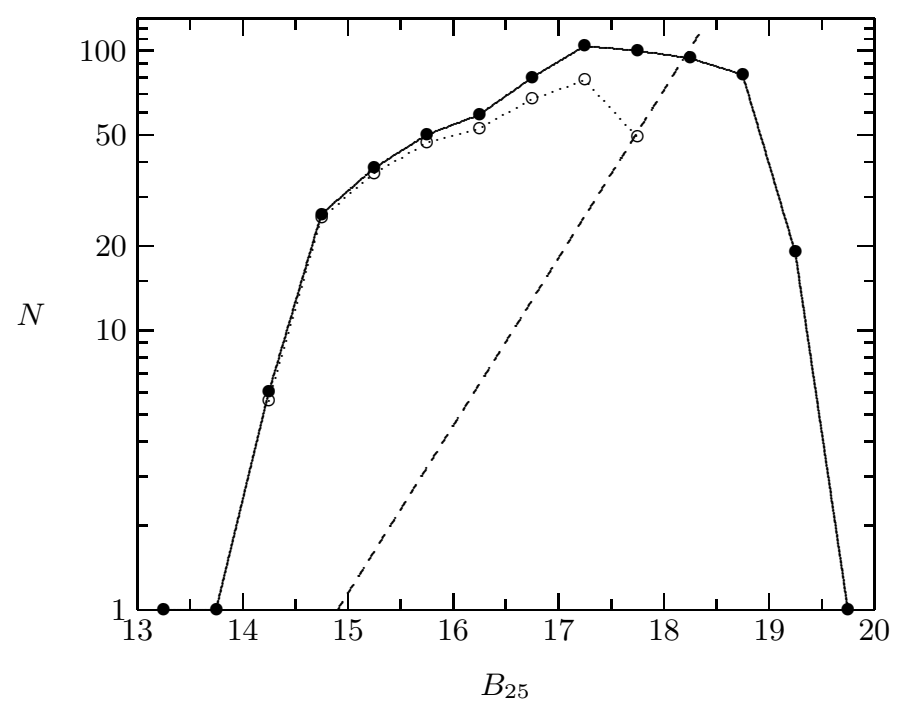

Fig. 12. Luminosity function of all catalogued galaxies in the field, i.e. number of galaxies $N$ vs. magnitude $B_{25}$ in $0.5 \mathrm{mag}$ bins. Filled circles: original data without correction for background contamination, open circles: background-corrected data. Straight, dashed line: number of background galaxies per 0.5 mag bin, derived from Eq. (2)

\subsection{Luminosity function}

The luminosity function (LF) for all galaxies is shown in Fig. 12, along with the number-magnitude relation for background galaxies (according to Eq.2) and the background-corrected LF. The uncertainties in the LF are dominated by the probably irregular Galactic foreground extinction and by background contamination at fainter magnitudes. For type-dependent LFs (Fig. 13), further uncertainties arise from the morphological classification. With regard to these uncertainties we have not tried to transform the measured $B_{25}$ magnitudes into $B_{\mathrm{T}}^{0}$ magnitudes.

Background contamination is expected to be unimportant for $B_{25}<17$. In the magnitude range $14.5<B_{25}<$ 17.5 , the LF is reasonably fitted by a power law with slope

$\alpha=\frac{\mathrm{d} \log N(L)}{\mathrm{d} \log L}=-\left(1+2.5 \frac{\mathrm{d} \log N(B)}{\mathrm{d} B}\right)$.

For the original data we have $\alpha_{\text {orig }}=-1.58 \pm 0.03$, whereas the corrected LF is slightly shallower with $\alpha_{\text {corr }}=$ $-1.47 \pm 0.04$. For the most luminous galaxies the LF is much steeper. The change of the slope appears near $B_{25}=14.7 \pm 0.5$ corresponding to $M_{B}=-21.3 \pm 0.5$ for a Hubble constant $H_{0}=50 \mathrm{~km} \mathrm{~s}^{-1} \mathrm{Mpc}^{-1}$. Within the uncertainties, this value agrees with the characteristic magnitude $M_{B_{\mathrm{T}}}^{*}$ given by Jerjen \& Tammann (1997) for the Virgo cluster.

It is more reasonable to consider the LFs for different morphological types (Binggeli et al. 1988; Jerjen et al. 1992; Jerjen \& Tammann 1997; Andreon 1998b). In Fig. 13, we show the results for A 426. The data 


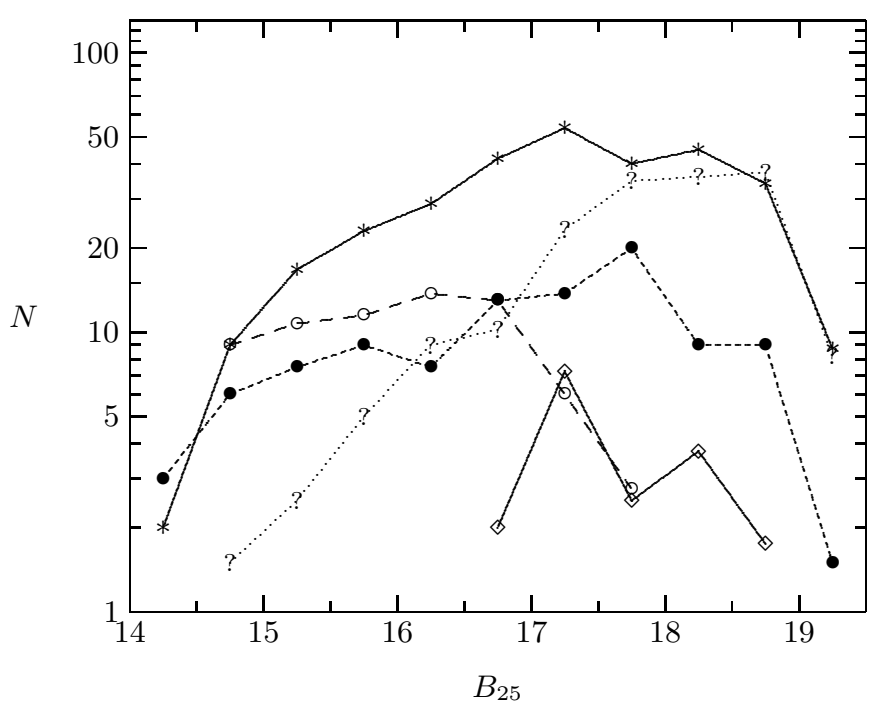

Fig. 13. Luminosity functions for different morphological types: $\mathrm{S}$ (solid, *), E (short dashes, •), S0 (long dashes, o), Irr (solid, $\diamond)$, non-classified (dotted, ?)

Table 4. Type-dependent LFs for spirals (S), irregulars (Irr), ellipticals (E), S0 (S0), and non-classified (?) galaxies. (Decadic logarithm of the number of galaxies per $0.5 \mathrm{mag}$ interval)

\begin{tabular}{cccccc}
\hline$B_{25}$ & $\mathrm{~S}$ & Irr & $\mathrm{E}$ & $\mathrm{S} 0$ & $?$ \\
\hline $14.0-14.5$ & 0.30 & 0.00 & 0.48 & 0.00 & 0.00 \\
$14.5-15.0$ & 0.95 & 0.00 & 0.78 & 0.95 & 0.18 \\
$15.0-15.5$ & 1.22 & 0.00 & 0.88 & 1.03 & 0.40 \\
$15.5-16.0$ & 1.36 & 0.00 & 0.95 & 1.06 & 0.70 \\
$16.0-16.5$ & 1.46 & 0.00 & 0.88 & 1.14 & 0.95 \\
$16.5-17.0$ & 1.62 & 0.30 & 1.11 & 1.12 & 1.01 \\
$17.0-17.5$ & 1.73 & 0.86 & 1.14 & 0.78 & 1.37 \\
$17.5-18.0$ & 1.60 & 0.40 & 1.30 & 0.44 & 1.54 \\
$18.0-18.5$ & 1.65 & 0.57 & 0.95 & 0.00 & 1.56 \\
$18.5-19.0$ & 1.53 & 0.24 & 0.95 & 0.00 & 1.57 \\
$19.0-19.5$ & 0.94 & 0.00 & 0.18 & 0.00 & 0.92 \\
\hline
\end{tabular}

are listed in Table 4. The counting convention from Sect. 7.5 has been used. We can compare Fig. 13 with the type-dependent composite LFs constructed by Andreon (1998b) from 5 clusters (Virgo, Fornax, Centaurus, Coma, Cl0939+4713). Firstly, we agree with Andreon that the LFs of Es, S0s and spirals are different but overlap largely in luminosity. Furthermore, Es show a rather broad distribution. On the other hand, we find disagreement with regard to S0s: from Andreon's data (his Table 1) we expect a strong peak in the S0 LF near $B_{25}=16.5$, where we indeed find the maximum, which is, however, much less pronounced than is expected from Andreon's LF. Nevertheless, S0s have the narrowest distribution both in Andreon's composite LF and in our A 426 data.

The most obvious feature in Fig. 13 is the strong increase in the spiral LF in the magnitude range where background contamination is not significant. For $15<B_{25} \leq$

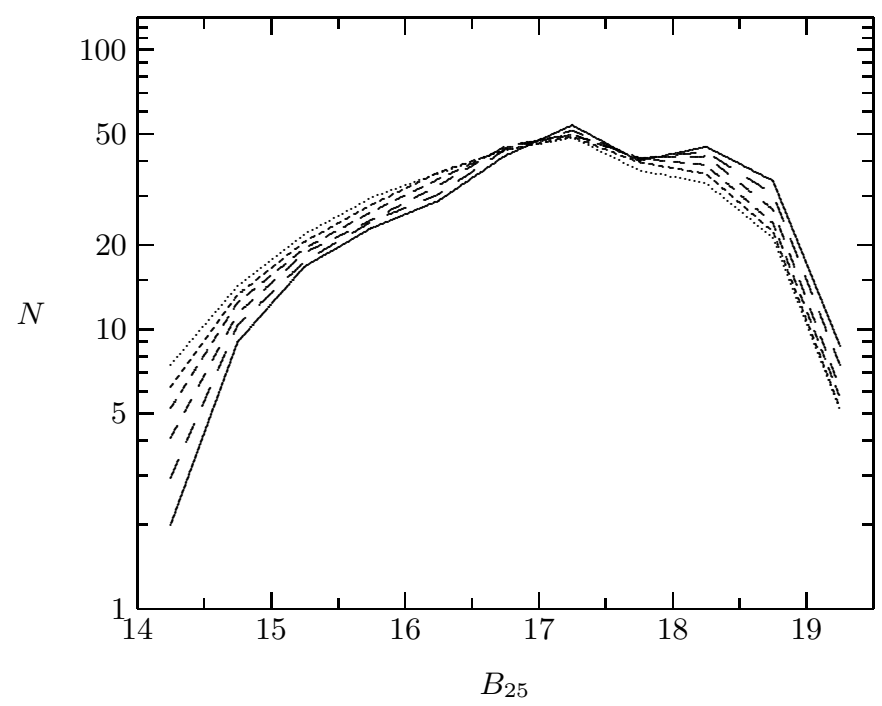

Fig. 14. Simulation of the effect of enhanced foreground extinction on the LF of spirals. For successively stronger extinction the observed LF (solid curve) corresponds to true, extinctioncorrected LFs (long dashes to dotted curves) with successively larger fractions of higher-luminosity spirals (see text)

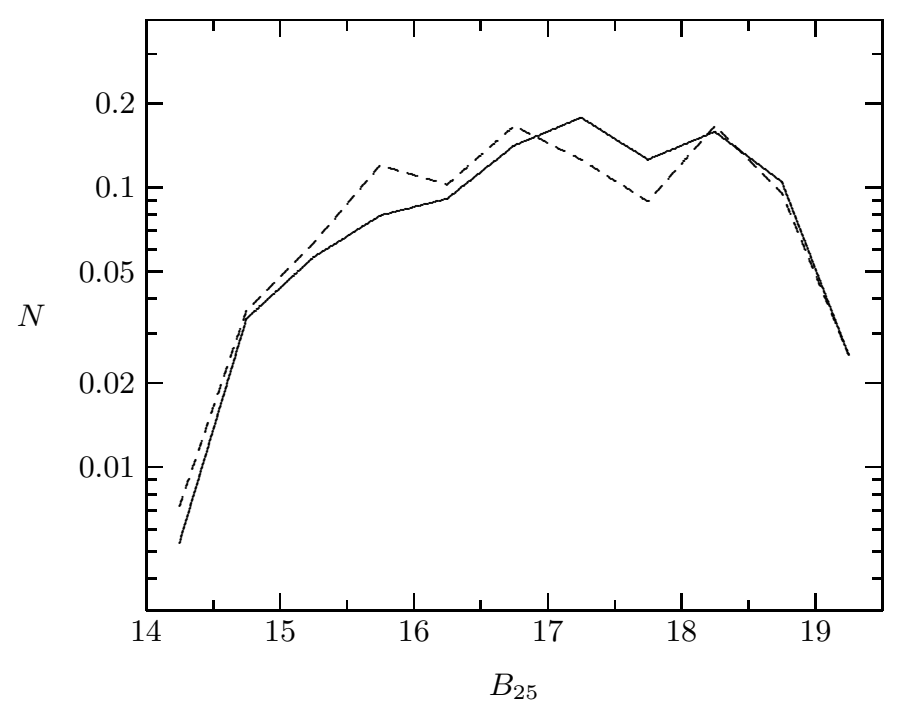

Fig. 15. Comparison of the LF for all spirals (solid) with the LF for spirals with projected cluster-centric distances $r \leq 50^{\prime}$ (dashed). The LFs are normalised to 1

17.5 we find $\alpha_{S}=-1.65 \pm 0.02$, i.e. steeper than the total LF. In the same magnitude range, the slope of the LF for all galaxies except spirals is only $\alpha_{\text {all }-\mathrm{S}} \leq 1.43 \pm 0.03$, even if we assume that there is no spiral among the unclassified galaxies. This result does not well agree with the assumption that different morphological types have the same LF.

One can argue that strong conclusions about the LFs are hampered by the possibility of irregular Galactic foreground extinction. This holds especially for the LF of spirals which are preferentially located in the external parts of the cluster, where extinction is largest (Sect. 7.1). The effect of foreground extinction on the spiral LF is 
essentially twofold. Firstly, the observed LF will become steeper than the true, extinction-corrected LF since the luminosities of the galaxies are under-estimated. For illustration, we performed Monte-Carlo simulations of the spiral LF using about $10^{5}$ galaxies. Starting with the observed LF from Fig. 13, we assume that a fraction $g$ of the spirals is affected by additional foreground extinction $\Delta A_{B}$ (in addition to the mean extinction $A_{B}=0.8$ adopted for the inner cluster region), where $\Delta A_{B}$ is randomly distributed in the interval $\left[0, \Delta A_{B, \max }\right]$. The corresponding extinction-corrected LFs are shown in Fig. 14 for $g=0.5$ and $\Delta A_{B, \max }=0.3,0.6,0.9,1.2$ and $1.5 \mathrm{mag}$, respectively. The interpretation of Fig. 14 is that uncorrected foreground extinction yields an apparent steepening of the observed LF near its maximum, i.e. at $B_{25} \approx 17$. The effect becomes stronger for higher values of $g$ and $\Delta A_{B \text {, max }}$, of course. Thus, we can not definitely exclude the possibility that the observed slope of the spiral LF is influenced by extinction. At the bright end $\left(B_{25} \lesssim 16\right)$, however, the slope is expected to be nearly unaffected. A quantitative correction of the LF can be made only on the basis of a detailed extinction map. Secondly, extinction may impair the visibility of the fainter outer part of spirals. Predominantly fainter objects will be either misclassified (for example as Es if only the brighter bulges are detected) or classified as unknown (? in Fig. 13) and will drop out of the statistics. As a consequence of this misclassification effect, the observed spiral LF is expected to be shallower than the true one.

To summarise, these both extinction effects work in opposite directions with regard to the observed slope of the spiral LF, and it is difficult to estimate the net effect. According to the IRAS $100 \mu \mathrm{m}$ map (see Ettori et al. 1998), the region with projected clustercentric distances $r<1^{\circ}$ seems significantly less affected by Galactic extinction than the more distant regions, in particular northeastward and south-westward, respectively. In Fig. 15, we show the LF for spirals with $r<50^{\prime}$ along with the total spiral LF from Fig. 13. The comparison yields no significant difference, though the trend shown in Fig. 14 is slightly indicated. The net effect of the extinction on the slope of the spiral LF for $B_{25} \lesssim 16$ seems to be negligible. Therefore, we guess that the stronger increase in the observed LF of spirals at brighter magnitudes, compared to other types, is probably real.

\subsection{Total luminosity of the Perseus cluster}

The combined apparent magnitudes $B_{25}$ of all galaxies in our survey yield a total magnitude $B_{\text {tot }}=9.44$. When we correct for background galaxies according to Eq. (2), the total luminosity is reduced by at most 0.14 mag. The contribution of non-cluster members to the total apparent luminosity is therefore $\leq 12 \%$. On the other hand, an unknown number of faint cluster galaxies is not included in our sample. A quick estimate shows that, under the assumption that the sample is essentially complete up to $B_{25}=18$, more than $10^{3}$ additional faint galaxies would be necessary to significantly alter the total luminosity. By applying galaxy luminosity functions from other studies of either field galaxies or cluster galaxies (Jerjen et al. 1992; Driver et al. 1995; De Propris \& Pritchet 1998), the contribution of missed faint galaxies to the total luminosity is estimated to be less than $0.1 \mathrm{mag}$.

From the total apparent luminosity $B_{\text {tot }}=$ $9.44 \pm 0.14$ combined with a distance modulus of $36.0 \mathrm{mag}-5 \log _{10} h_{50}$ (assuming $A_{B}=0.8 \mathrm{mag}, \mathrm{KS} 83$ ), the total $B$-band luminosity of the Perseus cluster galaxies within the central 10 square-degree is

$L_{B, \text { tot }}=(6.5 \pm 0.9) 10^{12} L_{B, \odot} \times h_{50}^{-2}$.

This value is more than twice as high as the Virgo Cluster luminosity (Sandage et al. 1985).

\section{Conclusions}

We present a homogenous catalogue of 660 galaxies brighter than $B_{25}=19.5$ in the central 10 square degrees area of the Perseus cluster based on a Schmidt plate survey. The catalogue provides accurate positions, $B_{25}$ magnitudes, position angles and major and minor axis of the $\mu_{B}=25 \mathrm{mag} \operatorname{arcsec}^{-2}$ isophote. Furthermore, morphological information is given, derived mainly from deep images obtained from the co-addition of a large number of digitised Schmidt plates. Cross-identifications and radial velocities are also listed if available. The galaxy catalogue is expected to be complete to $B_{25} \approx 18$. Background contamination may be important for $B_{25}>18$ but should be negligible for $B_{25}<17$.

We find a total fraction of about $50 \%$ spiral galaxies if we consider the whole field. On the other hand, the galaxies in the inner part $\left(r \lesssim 30^{\prime}\right)$ of the cluster are strongly dominated by types $\mathrm{E}$ and S0. In this context, we note that the BGP galaxy sample, used in several previous studies of the Perseus cluster, is limited to the central $30^{\prime}$ and is biased, therefore, towards E and S0 types. For example, KS 83 studied the BGP sample and found a significant spiral under-abundance in the Perseus cluster.

The investigation of the projected distribution of the different morphological types yields a strong radial segregation, density segregation, and segregation due to a privileged direction in the distribution of $\mathrm{E}+\mathrm{S} 0$ galaxies. All detected segregations are statistically significant. In agreement with Andreon (1994) we conclude that the Perseus cluster is not in a virialized state. The privileged direction is aligned with the elongation of the X-ray contours. In the region of the eastern X-ray excess (e.g., Ettori et al. 1998), we find a significantly higher fraction of spiral and irregular galaxies compared with the morphological mix of the galaxies on the opposite side of the X-ray centre. 
We detected a conspicuous clump in the projected distribution of the fainter $(B>16)$ galaxies at $\alpha(\mathrm{J} 2000.0)=$ $3^{\mathrm{h}} 20 \mathrm{~m}^{\mathrm{m}} 4, \delta(\mathrm{J} 2000.0)=43^{\circ} 4^{\prime}$, i.e. about $1.5^{\circ}$ north of the cluster centre, which is shown to be not a substructure of A 426. Radial velocities obtained for three galaxies of that clump indicate the presence of a background cluster at $z=0.050$. Owing to a probably patchy foreground extinction over the field we have not investigated other substructures in the projected galaxy distribution.

It seems not possible to define a unique centre for the projected galaxy distribution. Different cluster centres have been found for S+Irr galaxies, on the one hand, and $\mathrm{E}+\mathrm{S} 0$, on the other hand, reflecting a morphological segregation. Moreover, the optical cluster centre position significantly depends on the considered sky area. In no case, however, the optical centre position is in coincidence with the X-ray centre.

We have studied the galaxy alignments by means of extensive statistical tests. The results show that there are no significant preferred directions in the distribution of the orientation angles PA.

The LFs for different morphological types clearly differ from each other. The type-dependent LFs show several properties in agreement with universal type-dependent LFs predicted by Andreon (1998b). On the other hand, there are remarkable differences. In particular, the spiral LF is rather steep and the S0 LF shows no pronounced maximum. The total $B$-band luminosity of A 426 is estimated to $L_{B \text {,tot }}=(6.5 \pm 0.9) \times 10^{12} L_{B, \odot} \times h_{50}^{-2}$.

Acknowledgements. We thank the anonymous referee for constructive comments and suggestions. J.B. acknowledges financial support from the Deutsche Forschungsgemeinschaft under grant ME 1350/3. This research has been made use of the NASA/IPAC Extragalactic Database (NED) which is operated by the Jet Propulsion Laboratory, California Institute of Technology, under contract with the National Aeronautics and Space Administration.

\section{References}

Abell G.O., Corwin H.G., Olowin R.P., 1989, ApJS 70, 1

Allen S.W., Fabian A.C., 1997, MNRAS 286, 583

Allen S.W., Fabian A.C., Johnstone R.M., Nulsen P.E.J., Edge A.C., 1992, MNRAS 254, 51

Andreon S., 1994, A\&A 284, 801

Andreon S., 1996, A\&A 314, 763

Andreon S., 1998a, in: A new vision of an old cluster: untangling Coma Berenices, A. Mazure et al. (eds.). World Scientific, Singapore, p. 99

Andreon S., 1998b, A\&A 336, 98

Andreon S., Davoust E., Poulain P., 1997a, A\&AS 126, 67

Andreon S., Davoust E., Heim T., 1997b, A\&A 323, 337

Andruk V., Kharchenko N., Schilbach E., 1994, Astron. Nachr. 315,431

Arp H.C., Madore B.F., 1987, A Catalogue of Southern Peculiar Galaxies and Assiciations. Univ. Press, Cambridge Baier F.W., 1979, Astron. Nachr. 300, 243
Baier F.W., 1984, Astron. Nachr. 305, 111

Barnes J.E., 1992, ApJ 393, 484

Bautz L.P., Morgan W.W., 1970, ApJ 162, L149

Beers T.C., Tonry J.L., 1986, ApJ 300, 557

Binggeli B., Sandage A., Tammann G.A., 1988, ARA\&A 26, 509

Bird C.M., 1994, AJ 107, 1637

Böhringer H., Voges W., Fabian A.C., Edge A.C., Neumann D.M., 1993, MNRAS 265, L25

Börngen F., Chatchikjan E., 1967, Astron. Nachr. 289, 253

Branduardi-Raymont G., Fabricant D., Feigelson E., et al., 1981, ApJ 248, 55

Brunzendorf J., Meusinger H., 1996, Mitt. Astron. Ges. 12, 221

Brunzendorf J., Meusinger H., 1998, in: The Message of the Angles- Astrometry from 1798 to 1998, P. Brosche, et al. (eds.). H. Deutsch, Frankfurt, p. 148

Bucknell M.J., Godwin J.G., Peach J.V., 1979, MNRAS 188, 579 (BGP)

Burbidge E.M., Burbidge G.R., 1965, ApJ 142, 1351

Burns J.O., Sulkanen M.E., Gisler G.R., Perley R.A., 1992, ApJ 388, L49

Capaccioli M., de Vaucouleurs G., 1983, ApJS 52, 465

Casertano S., Hut P., 1985, ApJ 298, 80

Caulet A., Woodgate B.E., Brown L.W., et al., 1992, ApJ 388, 301

Chincarini G., Rood H., 1971, ApJ 168, 321

De Propris R., Pritchet C.J., 1998, AJ 116, 1118

Dixon W.V.D., Davidson A.F., Ferguson H.C., 1996, AJ 111, 130

Djorgovski S., 1987, in: Nearly Normal Galaxies, S.M. Faber (ed.). Springer, New York, p. 227

Doi M., Fukugita M., Okamure S., Tarusawa K., 1995, ApJS 97, 77

Dressler A., 1980, ApJ 236, 351

Dressler A., Shectman S.A., 1988, AJ 95, 985

Driver S.P., Windhorst R.A., Ostrander E.J., 1995, ApJ 449, L23

Escalara E., Biviano A., Girardi M., et al., 1994, ApJ 423, 539

Ettori S., Fabian A.C., White D.A., 1998, MNRAS 300, 837

Fabian A.C., 1994, ARA\&A 32, 277

Fabian A.C., Nulsen P.E.J., 1977, MNRAS 180, 479

Ferruit P., Pécontal E., 1994, A\&A 288, 65

Ferruit P., Adam G., Binette L., Pécontal E., 1997, New Astronomy 2, 345

Fitchett M.J., Webster R.L., 1987, ApJ 317, 653

Geller M.J., Beers T.C., 1982, PASP 94, 421

Gregory S.A., Thompson L.A., Tifft W.G., 1981, ApJ 243, 411

Hambly N.C., Miller L., MacGillivray H.T., Herd J.T., Cormack W.A., 1998, MNRAS 298, 897

Holtzman J.A., Faber S.M., Shaya E.J., et al., 1992, AJ 103, 691

Howard S., Keel W.C., Byrd G., Burkey J., 1993, ApJ 417, 502

Hu F.X., Wu G.X., Su H.J., Liu Y.Z., 1995, A\&A 302, 45

Jerjen H., Tammann G.A., Binggeli B., 1992, in: Morphological and Physical Classification of Galaxies, Longo G. et al. (eds.). Kluwer, Dordrecht, p. 17

Jerjen H., Tammann G.A., 1997, A\&A 321, 713

Karachentsev I.D., 1972, Soobshch. Spets. Astrofiz. Obs 1, 3

Kemp S.N., Meaburn J., 1995, in: The Future Utilisation of Schmidt Telescopes, Chapman J.M. et al. (eds.), ASP Conf. Ser. 84, 200 
Kent S.M., Sargent W.L.W., 1979, ApJ 230, 667

Kent S.M., Sargent W.L.W., 1983, AJ 88, 697 (KS83)

Klemola A.R., Jones B.F., Hanson R.B., 1987, AJ 94, 501

Lehmann H., Häupl W., 1988, in: Astrophotography, Marx S. (ed.). Springer Berlin, p. 64

Malin D.F., 1994, in: Astronomy from Wide-Field Imaging. IAU Symp. 161, McGillivray H.T. et al.. Kluwer, Dordrecht, p. 567

Malin D.F., Carter D., 1993, ApJ 274, 534

McNamara B.R., O'Connell R.W., Sarazin G.L., 1996, AJ 112, 91

Melnick J., Sargent W.L.W., 1977, AJ 215, 401

Meusinger H., Brunzendorf J., 1996, in: Röntgenstrahlung from the Universe, Zimmermann H.U., Trümper J.E., Yorke H. (eds.), MPE Report 263, p. 599

Meusinger H., Brunzendorf J., Pollas C., Szécsényi-Nagy G., Turatto M., 1999, in: Treasure Hunting in Astronomical Plate Archives, Kroll P., et al. (eds.). Deutsch H., Frankfurt (in press)

Meusinger H., Brunzendorf J., Scholz R.-D., Irwin M., 1999, in: Treasure Hunting in Astronomical Plate Archives, Kroll P. et al. (eds.). Deutsch H., Frankfurt (in press)

Miller L., Cormack W., Paterson M., Beard S., Lawrence L., 1992, in: Digitised Optical Sky Surveys, MacGillivray H.T., Thomson E.B. (eds.). Dordrecht, Kluwer, p. 133

Minkowski R., 1957, in: Radio Astronomy, IAU Symp. 4, van de Hulst H.C. (ed.). Cambridge, University Press, p. 107

Mohr J.J., Fabricant D.G., Geller M.J., 1993, ApJ 413, 492

Mushotsky R.F., Serlemitsos P.J., Smith B.W., Bolt E.A., Holt S.S., 1978, ApJ 225, 21

Naim A., Ratnatunga K.U., Griffiths R.E., 1997, ApJ 476, 510

Nulsen P.E.J., Fabian A.C., 1980, MNRAS 191, 887

Okamura S., 1988, PASP 100, 524

O'Neil K., Bothun G.D., Cornell M.E., 1997, AJ 113, 1212

Paturel G., Fouqué P., Botinelli L., Gouguenheim L., 1989, A\&AS 80, 299

Paturel G., Petit C., Botinelli L., Fouqué P., Gouguenheim L., 1993, Bull. Inf. CDS 43
Pedlar A., Ghataure H.S., Davies R.D., et al., 1990, MNRAS 246, 477

Peres C.B., Fabian A.C., Edge A.C., Allen S.W., Johnstone R.M., White D.A., 1998, MNRAS 298, 416

Poulain P, Nieto J.-L., Davoust E., 1992, A\&AS 95, 129

Röser S., Bastian U., 1991, PPM Star Catalogue 1,2. Spektrum Akademischer Verlag, Heidelberg, Berlin, New York

Rubin V.C., Ford Jr. W.K., Peterson C.J., Oort J.H., 1977, ApJ 211, 693

Ryle M., Windram M.D., 1968, MNRAS 138, 1

Sandage A., Binggeli B., Tammann G.A., 1985, AJ 90, 1759

Sanromà M., Salvador-Soé E., 1990, ApJ 360, 16

Schwarz R.A., Edge A.C., Voges W., Böhringer H., Ebeling H., Briel U.G., 1992, A\&A 256, L11

Schweizer F., Seitzer P., 1988, ApJ 328, 88

Siegel S., Castellan N.J., 1988, Nonparametric Statistics for the Behavioral Sciences. McGraw-Hill, New York

Snyder W.A., Kowalski M.P., Cruddace R.G., et al., 1990, ApJ 365, 460

Strom S.E., Strom K.M., 1978, AJ 83, 732

Struble M.F., Rood H.J., 1987, ApJS 63, 555

Ulmer M.P., Cruddace R.G., Wood K., et al., 1980, ApJ 236, 58

Ulmer M.P., Wirth G.D., Kowalski M.P., 1992, ApJ 397, 430

van den Bergh S., 1964, AJ 69, 610

Vermeulen R.C., Readhead A.C.S., Backer D.C., 1994, ApJ 430, L41

Véron P., 1978, Nat 272, 430

Weedman D.W., 1975, ApJ 195, 587

Whitmore B.C., Lucas R.A., McElroy D.M., et al., 1990, AJ 100,1489

Whitmore B.C., Gilmore D.M., Jones C., 1993, ApJ 407, 489

Wu G.X., Hu F.X., Su H.J., Liu Y.Z., 1997, A\&A 323, 317

Young C.K., Currie M.J., 1998, A\&AS 127, 367

Zwicky F., Kowal C.T., 1968, Catalog of Galaxies and Clusters of Galaxies, California Institute of Technology, Pasadena, Vol. VI 